\title{
Quantifying seismic anisotropy induced by small-scale chemical heterogeneities
}

\author{
C. Alder, ${ }^{1}$ T. Bodin, ${ }^{1}$ Y. Ricard,${ }^{1}$ Y. Capdeville, ${ }^{2}$ E. Debayle ${ }^{1}$ and J.P. Montagner ${ }^{3}$ \\ ${ }^{1}$ Univ Lyon, Université Lyon 1, ENS de Lyon, CNRS, UMR 5276 LGL-TPE, F-69622, Villeurbanne, France. E-mail: chloe.alder@ens-lyon.fr \\ ${ }^{2}$ Laboratoire de Planétologie et Géodynamique, CNRS, UMR 6112, Université de Nantes, F-44322 Nantex Cedex 3, France \\ ${ }^{3}$ Équipe de Sismologie, Institut de Physique du Globe de Paris, CNRS, UMR 7154, F-75238 Paris Cedex 05, France
}

Accepted 2017 September 18. Received 2017 August 3; in original form 2017 April 4

\begin{abstract}
S UMMAR Y
Observations of seismic anisotropy are usually used as a proxy for lattice-preferred orientation (LPO) of anisotropic minerals in the Earth's mantle. In this way, seismic anisotropy observed in tomographic models provides important constraints on the geometry of mantle deformation associated with thermal convection and plate tectonics. However, in addition to LPO, smallscale heterogeneities that cannot be resolved by long-period seismic waves may also produce anisotropy. The observed (i.e. apparent) anisotropy is then a combination of an intrinsic and an extrinsic component. Assuming the Earth's mantle exhibits petrological inhomogeneities at all scales, tomographic models built from long-period seismic waves may thus display extrinsic anisotropy. In this paper, we investigate the relation between the amplitude of seismic heterogeneities and the level of induced $S$-wave radial anisotropy as seen by long-period seismic waves. We generate some simple 1-D and 2-D isotropic models that exhibit a power spectrum of heterogeneities as what is expected for the Earth's mantle, that is, varying as $1 / k$, with $k$ the wavenumber of these heterogeneities. The 1-D toy models correspond to simple layered media. In the 2-D case, our models depict marble-cake patterns in which an anomaly in shear wave velocity has been advected within convective cells. The long-wavelength equivalents of these models are computed using upscaling relations that link properties of a rapidly varying elastic medium to properties of the effective, that is, apparent, medium as seen by long-period waves. The resulting homogenized media exhibit extrinsic anisotropy and represent what would be observed in tomography. In the 1-D case, we analytically show that the level of anisotropy increases with the square of the amplitude of heterogeneities. This relation is numerically verified for both 1-D and 2-D media. In addition, we predict that 10 per cent of chemical heterogeneities in 2-D marble-cake models can induce more than 3.9 percent of extrinsic radial $S$-wave anisotropy. We thus predict that a non-negligible part of the observed anisotropy in tomographic models may be the result of unmapped small-scale heterogeneities in the mantle, mainly in the form of fine layering, and that caution should be taken when interpreting observed anisotropy in terms of LPO and mantle deformation. This effect may be particularly strong in the lithosphere where chemical heterogeneities are assumed to be the strongest.
\end{abstract}

Key words: Seismic anisotropy; Seismic tomography; Surface waves and free oscillations.

\section{INTRODUCTION}

For many years, seismologists have been mapping the Earth from seismic waves. For practical reasons, the first Earth-like models were isotropic and consisted of concentric homogeneous shells (see Montagner 1998, for a review). Yet, over the past several decades, multiple studies have established that seismic anisotropy is needed to explain seismic observations, such as the Rayleigh-Love discrepancy (Anderson 1961); the azimuthal dependence of body-wave velocity under the ocean (Hess 1964); or the shear wave splitting (Vinnik et al. 1989).
Seismic anisotropy can be caused by many physical processes and is observed at different scales. At the crystal scale, olivine, the most abundant mineral in the upper mantle, exhibits an orthorhombic symmetry. This specific symmetry leads, for a single crystal, to seismic anisotropy with fast and slow velocities differing by more than 20 per cent for $P$-waves (Mainprice et al. 2000; Mao et al. 2015). Other important mantle constituents such as pyroxenes are anisotropic as well: around 15 percent for orthopyroxene and 25 per cent for clinopyroxene (Mainprice et al. 2000). However, as olivine is far more abundant than pyroxene in the mantle peridotite, it is thought to be a dominant element in the bulk anisotropy at large 
scale (Babuška \& Cara 1991). Laboratory experiments and field observations on ophiolites show that under finite strain accumulation, a preferential orientation of these minerals' crystalline lattices can arise from plastic deformation. This process is usually referred to as lattice-preferred orientation (LPO) - or crystallographic-preferred orientation (CPO). Assuming the deformation due to mantle convection is coherent over large spatial scales, LPO is considered to be at the origin of the long-wavelength anisotropy observed in the upper mantle. Therefore, anisotropy in upper mantle tomographic models is generally assumed to be primarily intrinsic (i.e. due to LPO) and is used as a powerful indicator of large-scale mantle deformation due to thermal convection and plate tectonics (Debayle \& Ricard 2013).

However, in addition to LPO, small-scale inhomogeneities that cannot be resolved by long-period seismic waves may also produce large-scale extrinsic anisotropy (Maupin \& Park 2007). For example, Backus (1962) showed that, when sampled by seismic waves with a minimum wavelength much larger than the average layer thickness, a stack of horizontal isotropic layers is equivalent to a homogeneous radially anisotropic medium. Therefore, whether a medium is heterogeneous or anisotropic is actually a matter of scale at which we analyse its properties (Maupin \& Park 2007). This has led seismologists to separately interpret different data types that sample the Earth at different scales, thus often producing incompatible models. Short-period converted and reflected body waves see sharp discontinuities interpreted in terms of chemical stratification (e.g. Tauzin et al. 2016; Hier-Majumder \& Tauzin 2017) or phase changes (e.g. Tauzin \& Ricard 2014), whereas tomographic models constructed from long-period surface waves depict a smooth anisotropic upper mantle and are usually interpreted in terms of mantle flow (see Bodin et al. 2015, for a review). However, sharp discontinuities that are not resolved in tomographic studies may also produce extrinsic anisotropy at large scale. In this way, the anisotropy observed in tomographic studies can be qualified as apparent anisotropy: it is thought to be a combination of both an intrinsic component due to LPO and an extrinsic part induced by small-scale isotropic heterogeneities, mainly in the form of fine layering (Backus 1962; Fichtner et al. 2013; Wang et al. 2013; Wang et al. 2015). A challenging issue is, therefore, to separate the extrinsic and intrinsic contributions to anisotropy, as they should be interpreted in terms of different physical processes. Unfortunately, the current state of available seismic data only provides weak constraints on these relative contributions (Fichtner et al. 2013) and seismic waves sampling either a heterogeneous or an anisotropic material may induce waveforms that are indistinguishable from one another (Levshin \& Ratnikova 1984).

The relation between small-scale isotropic inhomogeneities and extrinsic anisotropy has been numerically tested in synthetic models. Maupin (2002) used a multiple-scattering scheme to model the propagation of surface waves in 3-D isotropic structures. Notice that she only considered inhomogeneities that were both locally isotropic and with an isotropic distribution (her approach precluded isotropic anomalies with an anisotropic distribution, such as laminations). She found that the discrepancy between Love and Rayleigh wave velocities varies linearly with the variance of small-scale velocity variations. She showed that the discrepancy obtained in this way is negligible compared to observations in some regions where it can reach up to 9 per cent. On the contrary, although Fichtner et al. (2013) and Wang et al. (2013, 2015) did not establish any mathematical relation between heterogeneities and anisotropy, they showed that the contribution of extrinsic anisotropy related to fine layering in the mantle may contribute in a non-negligible way to the observed anisotropy.
Our goal here is to numerically and analytically evaluate the relation between the level of heterogeneities and that of extrinsic anisotropy in simple isotropic media. This relation may be useful to better interpret anisotropy in tomographic models. We chose to limit our study to the case of $S$-wave radial anisotropy. In Section 2, we discuss the power spectrum of petrological heterogeneities in the Earth's mantle, as well as the size and amplitude of these inhomogeneities. Section 3 is an overview of homogenization methods that allow to compute the effective medium corresponding to an initial small-scale medium, that is, its long-wavelength equivalent. Section 4 presents how we built simple 1-D isotropic layered media with a $1 / k$ power spectrum, as expected for the mantle. In this section, we analytically prove that the extrinsic anisotropy in the long-wavelength equivalent medium increases as the square of heterogeneities present in the initial isotropic small-scale medium. Using explicit upscaling relations linking properties of the finely layered media to properties of their long-wavelength equivalents (Backus 1962), we were able to numerically verify this relation. In Section 5, we extend our study to 2-D marble-cake models, where chemical heterogeneities are stirred in a convective fluid. The longwavelength equivalents of these 2-D models are computed using the Fast Fourier Homogenization technique (Capdeville et al. 2015). Here, we also find that the level of extrinsic anisotropy varies as the square of the heterogeneities. In addition, we find that 10 per cent of chemical heterogeneities in the 2-D marble-cake models can induce more than 3.9 per cent of extrinsic radial $S$-wave anisotropy. These results suggest that a non-negligible part of the observed anisotropy in tomographic models may be due to unmapped heterogeneities, mainly in the form of fine layering.

\section{HETEROGENEITIES IN THE EARTH'S MANTLE}

Heterogeneities exist at different scales in the mantle. Thermal convection is responsible for smooth lateral variations in temperature resulting in large-scale heterogeneities. Since the chemical diffusivity of mantle materials is low compared to thermal diffusivity (Farber et al. 1994; Yamazaki et al. 2000) small-scale inhomogeneities are on the contrary more likely to be due to chemical or petrological anomalies (Xu et al. 2008; Ricard et al. 2014; Mancinelli et al. 2016). The most plausible origin of these chemical heterogeneities is the constant injection of oceanic lithosphere into the mantle at subduction zones (Coltice \& Ricard 1999). In the 1980 s, geochemical observations of elementary fractionation between the crust and the upper mantle, as well as structural studies of peridotitic massifs, led Allegre \& Turcotte (1986) to propose a geodynamic model that would result in a marble-cake mantle structure. In this model, the subducted oceanic lithosphere is stretched and thinned by the normal and shear strains in the convecting mantle. In this process, the spatial wavelength of heterogeneities decreases progressively and the resulting strips are eventually destroyed, either by being reprocessed at oceanic ridges or by dissolution processes at decimetric scales.

Following the pioneering work of Batchelor (1959), Olson et al. (1984) and Antonsen \& Ott (1991) showed that when heterogeneities are continuously injected into a chaotic convective fluid and multiply folded, their power spectrum should vary as $1 / k$ where $k$ is the wavenumber of the heterogeneities $(k=2 \pi / \lambda$ with $\lambda$ the spatial wavelength). The mantle is therefore believed to have such a power spectrum and is said to be scale-invariant or self-similar. This type of power spectrum has been documented on different data types and at different scales. In geochemistry, Agranier et al. (2005) 
observed in isotopic variations of mid-ocean ridge basalts a clear $1 / k$ spectrum along much of the Atlantic ridge. In seismology, the long-wavelength structure of tomographic models also shows a $1 / k$ structure. Kennett \& Furumura (2013) studied the high-frequency coda of $P$ and $S$ body waves recorded at ocean bottom seismometers. They found that these seismic records are consistent with a quasi-laminated oceanic lithosphere with heterogeneities showing much longer horizontal $(\sim 10 \mathrm{~km})$ than vertical $(\sim 0.5 \mathrm{~km})$ correlation lengths, which is in agreement with the laminated structure of the mantle invoked in the marble-cake model of Allegre \& Turcotte (1986). Recently, Mancinelli et al. (2016) used observations from high-frequency scattering, long-period scattering, and tomography to constrain the spectrum of the upper mantle. They showed that a self-similar mixture of basalt and harzburgite can explain the large wavenumber content of a $1 / k$ power spectrum for the mantle.

Up to now, scientists have not reached a consensus regarding the frequency band where this spectrum exists, that is, the minimum and maximum wavelengths of petrological heterogeneities in the mantle. The thickness of oceanic slabs subducting into the mantle is about $7 \mathrm{~km}$ for the basaltic crust and $100 \mathrm{~km}$ for the whole lithosphere. The folding and thickening of slabs in the lower mantle (Ricard et al. 1993; Ribe et al. 2007) and their accumulation at the base of the mantle may contribute to produce larger scale anomalies such as the strong degrees 2 and 3 at the base of the mantle (e.g. Durand et al. 2016). Regarding the highest wavenumber content of the spectrum, the mixture of basalt and harzburgite in Mancinelli et al. (2016) explains the observed power-spectrum for wavelengths down to $1 \mathrm{~km}$. However, several studies based on field observations and mixing modelling argued that the mantle is inhomogeneous down to the centimetre scale (Hoffman \& McKenzie 1985; Kellogg \& Turcotte 1987; Farber et al. 1994). Below this size of heterogeneities, chemical diffusion is expected to rapidly homogenize the material.

According to the marble-cake model of Allegre \& Turcotte (1986), the oceanic lithosphere composed of a basaltic crust and harzburgitic mantle is mixed within an undifferentiated mantle. In this context, numerous geodynamical and geochemical studies suggest that the upper mantle is a mechanical mixture of two endmembers, which are harzburgite and basalt, the latter becoming eclogite at high pressure (Hofmann 1988; Christensen \& Hofmann 1994; Morgan \& Morgan 1999; Nakagawa \& Buffett 2005; Brandenburg \& van Keken 2007; Xu et al. 2008; Nakagawa \& Tackley 2014; Ballmer et al. 2015; Mancinelli et al. 2016). Assuming the lithological integrity of these two end-members is preserved with depth and over significant geological times, the mixture of basalt and harzburgite is responsible for large and localized seismic velocity contrasts in the mantle (Stixrude \& Jeanloz 2007; Xu et al. 2008).

Based on these conclusions, in this paper we consider the mantle as a binary system in which the level of heterogeneities is controlled by the shear wave velocity contrast between two end-members having a different composition (e.g. basalt and harzburgite). This level of heterogeneities will hereafter be expressed as the amplitude of $S$-wave velocity variations in the mantle around a mean value, $V_{0}$. It will be referred to as

$\frac{\mathrm{d} V}{V_{0}}=\frac{V_{s_{\max }}-V_{s_{\min }}}{V_{s_{\max }}+V_{s_{\min }}}$

where $\mathrm{d} V=\left(V_{s_{\max }}-V_{s_{\min }}\right) / 2$ and $V_{0}=\left(V_{s_{\max }}+V_{s_{\min }}\right) / 2$, with respectively $V_{s_{\max }}$ and $V_{s_{\min }}$ the maximum and minimum shear wave velocity in the binary system. Note that in the hypothetical case where the proportions of the two end-members are the same, $\mathrm{d} V / V_{0}$ corresponds to $\sigma_{V} / V_{0}$, the standard deviation of $V_{s}$ over the mean.

This contrast of velocity $\mathrm{d} V / V_{0}$, between basalt and harzburgite, is the strongest in the lithosphere where it can reach about 10 per cent (e.g. Stixrude \& Jeanloz 2007; Xu et al. 2008). In the rest of the upper mantle, from the asthenospheric low-velocity zone to the $410 \mathrm{~km}$ discontinuity, $\mathrm{d} V / V_{0}$ is less important but still corresponds to about 5 per cent of heterogeneities. In the lower mantle, seismic velocities are less well constrained but it is assumed that $1-2$ per cent of petrological heterogeneities remain down to the base of the mantle (Ricard et al. 2005).

\section{THE ELASTIC HOMOGENIZATION}

Homogenization, effective media or upscaling techniques gather a wide range of methods able to compute effective properties and equations of a fine-scale problem when large scale properties are needed. In the context of wave propagation, the idea is to remove the heterogeneities of scale much smaller than the minimum wavelength of the wavefield and to replace them by effective properties. For long-period elastic waves propagating in stratified media, Backus (1962) gave explicit formulas to upscale finely layered media. For periodic media, an important class of methods, the two-scale homogenization methods, have been developed (e.g. Sanchez-Palencia 1980). To obtain the effective media, the effective equations, and local correctors, two-scale homogenization methods require solving the so-called periodic-cell problem. This periodic-cell problem can be solved analytically only for the specific case of layered media, whereas a numerical method such as finite elements is necessary for more general media. For stochastic media, methods formally similar to the two-scale homogenization methods exist (e.g. Bensoussan et al. 1978; Blanc et al. 2007). Typical geological media present no spatial periodicity, no natural scale separation or any kind of spatial statistical invariance. This difficulty excludes all of the above mentioned homogenization techniques to upscale geological media. To fill this gap, the non-periodic homogenization technique (Capdeville et al. 2010a,b; Guillot et al. 2010; Capdeville \& Cance 2015) has recently been introduced. So far, the non-periodic scale homogenization theory is mostly used as a pre-processing step making it possible to simplify complex media, allowing then to avoid complex meshes and leading to a quicker solution to compute synthetic seismograms.

Our goal here is different. Assuming a perfect data coverage, Capdeville et al. (2013) showed in the 1-D case that a tomographic model is a homogenized model. The elastic homogenization can therefore be seen as a 'tomographic operator' since it provides the best image that one would get from a tomographic inversion (because data coverage is never perfect, a tomography can only be a degraded version of the homogenized image). Under this assumption, in this paper we use Backus' homogenization (Backus 1962) to retrieve the best tomographic equivalent of layered media built in agreement with the marble-cake model of Allegre \& Turcotte (1986). Assuming the 'tomographic operator' hypothesis is still valid for 2-D media, we use the Fast Fourier Homogenization developed by Capdeville et al. (2015) to numerically mimic how 2-D marble-cake patterns would be seen by tomography. 


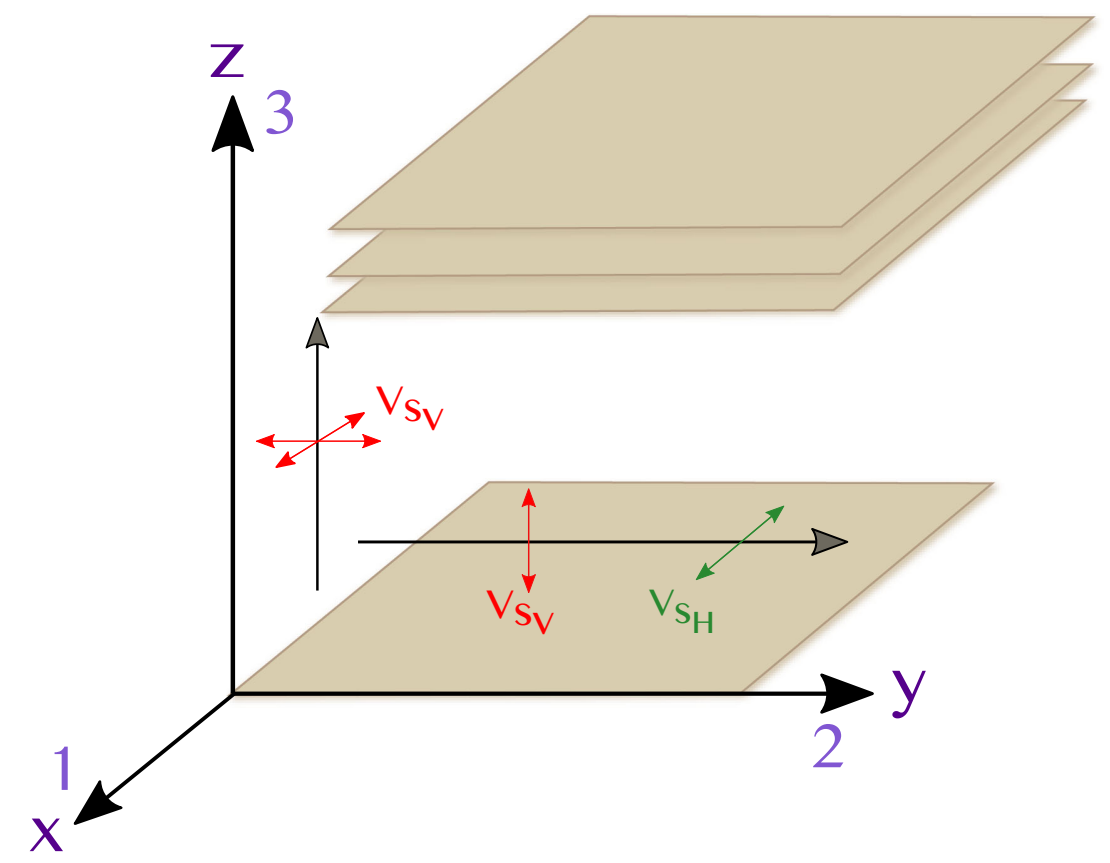

Figure 1. Coordinate system in the 1-D layered case where black arrows are the direction of propagation whereas coloured arrows correspond to polarization directions. $V_{S H}$ is the velocity of a horizontally propagating $S$-wave with horizontal polarization, $V_{S V}$ corresponds either to the velocity of a horizontally propagating $S$-wave with vertical polarization or to a vertically propagating wave with horizontal polarization.

\section{EXTRINSIC ANISOTROPY IN 1-D ISOTROPIC LAYERED MEDIA}

\subsection{Creating 1-D velocity models with a $1 / k$ power spectrum}

In this section, we consider a stack of planar layers (see Fig. 1), where each layer is isotropic and with a velocity that can only take one of two different values (either $V_{s_{\max }}$ or $V_{s_{\min }}$ ). In addition, we impose the corresponding signal to have a $1 / k$ power spectrum. To do so, we build a square signal following the alternating fractal renewable process described in Lowen \& Teich (2005), where the interval between discontinuities, $\lambda$, follows a decaying power-law probability density function $p$ such as:

$p(\lambda)=\frac{\gamma}{\lambda_{\min }^{-\gamma}-\lambda_{\max }^{-\gamma}} \times \begin{cases}\lambda^{-(\gamma+1)} & \text { if } \lambda_{\min }<\lambda<\lambda_{\max } \\ 0 & \text { otherwise }\end{cases}$

with respectively, $\lambda_{\min }$ and $\lambda_{\max }$ the minimum and maximum spatial wavelengths of the signal. For $\gamma=1$ in (2), this produces a binary medium with a power spectrum varying as $1 / k$ in the interval $\left[\lambda_{\min }, \lambda_{\max }\right]$. For wavelengths larger than $\lambda_{\max }$, the power spectrum is flat, that is, it is a white spectrum, whereas it decreases as $1 / k^{2}$ for wavelengths smaller than $\lambda_{\min }$. The resulting signal and the associated power spectrum are depicted in Fig. 2 where we chose arbitrarily $\lambda_{\max }=H$, where $H=1000 \mathrm{~km}$, the total length of the signal, and $\lambda_{\min }=2 \delta z$, where $\delta z$ is the spatial resolution in depth, which is equal to $250 \mathrm{~m}$. In the example displayed in Fig. 2, the medium was assigned a maximum shear wave velocity $V_{S_{\max }}$ of $4.5 \mathrm{~km} \mathrm{~s}^{-1}$ and exhibits 5 per cent of heterogeneities, that is, $\mathrm{d} V / V_{0}$ $=0.05$ in eq. (1).

\subsection{The Backus' homogenization}

Our stack of layers presents a cylindrical, that is, hexagonal, symmetry with an axis of symmetry assumed to be the vertical axis (axis $z$ or 3 in Fig. 1) and is usually referred to as a vertical transversely isotropic medium or VTI medium. Such a medium can be described by the 5 Love parameters: $A, C, F, L, N$ (Love 1927), with $L=N=\mu$, the rigidity modulus in the isotropic case. When the vertical axis is along axis 3 , the local elastic tensor $C_{i j}$ in Kelvin notation (Thomson 1856, 1878; Helbig 1994; Dellinger et al. 1998) is expressed as follows:

$C_{i j}=\left(\begin{array}{cccccc}A & A-2 N & F & 0 & 0 & 0 \\ A-2 N & A & F & 0 & 0 & 0 \\ F & F & C & 0 & 0 & 0 \\ 0 & 0 & 0 & 2 L & 0 & 0 \\ 0 & 0 & 0 & 0 & 2 L & 0 \\ 0 & 0 & 0 & 0 & 0 & 2 N\end{array}\right)$

To express the fourth-order elastic tensor $C_{i j k l}$ from Hooke's law as the $C_{i j} 6 \times 6$ matrix in (3), Kelvin notation follows the same symmetry-based convention as the more traditional Voigt notation (e.g. Maupin \& Park 2007). However, this notation enables to express the $C_{i j k l}$ elastic tensor as $C_{i j}$ without losing the tensor property (see Dellinger et al. 1998, for a review).

For weak anisotropy, shear waves are primarily sensitive to the two parameters $N$ and $L$ following the relations:

$V_{S H}=\sqrt{N / \rho}$

$V_{S V}=\sqrt{L / \rho}$

where $\rho$ is the density, and $V_{S H}$ and $V_{S V}$ are respectively the velocities for $S H$ and $S V$ waves, as described in Fig. 1 (Anderson 1961; Babuška \& Cara 1991). In this case, the level of radial anisotropy is usually expressed by the $\xi$ parameter (Anderson 1961) where:

$\xi=\frac{V_{S H}^{2}}{V_{S V}^{2}}=\frac{N}{L}$ 

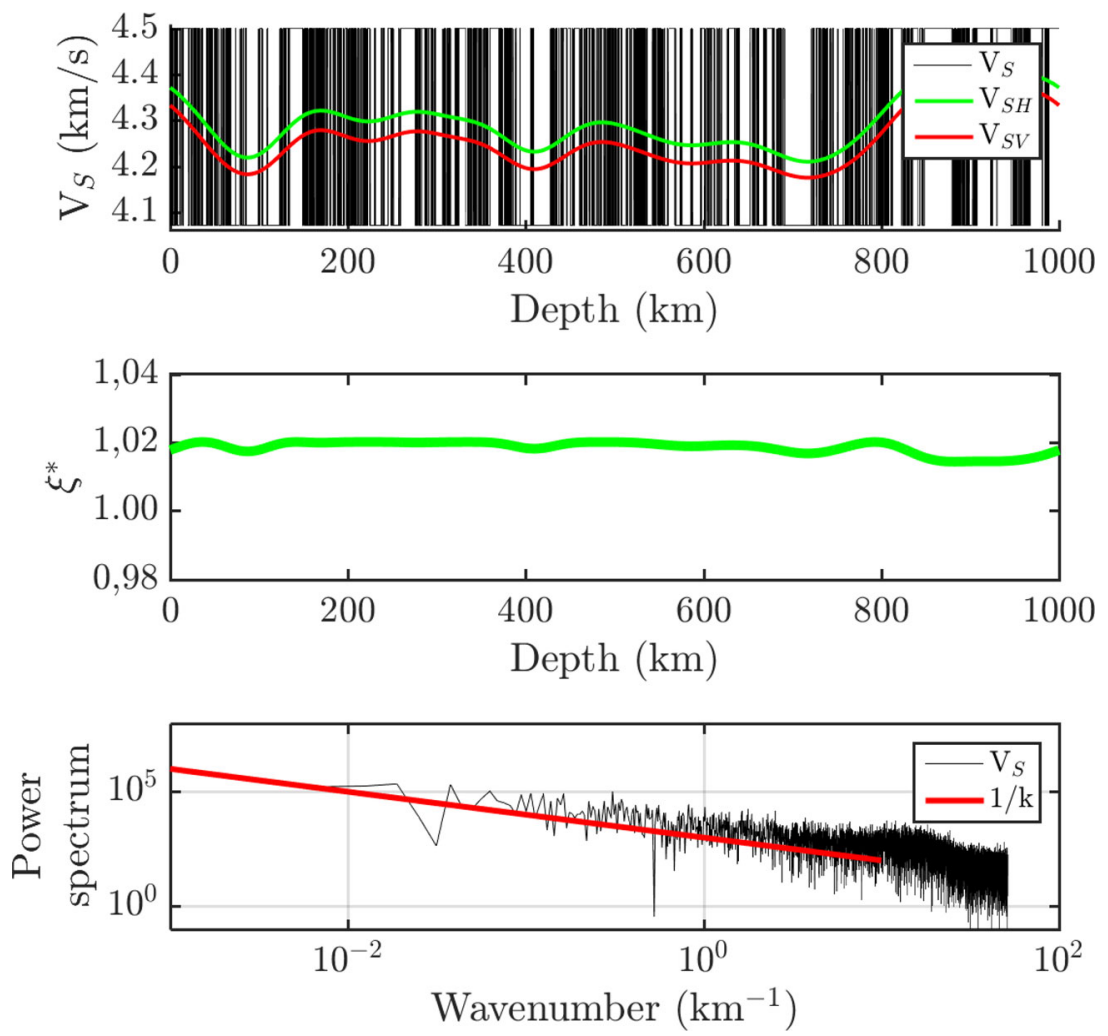

Figure 2. Top panel: the isotropic $V_{s}$ signal in black, corresponding to a finely layered medium with 5 per cent of heterogeneities (i.e. $\mathrm{d} V / V_{0}=0.05$ ), is homogenized for a wavelength $\lambda_{h}=200 \mathrm{~km}$. This homogenized, that is, effective or upscaled medium is the long-wavelength equivalent of the isotropic small-scale medium. It is described by $V_{S H}$ in green and $V_{S V}$ in red (see Fig. 1 for the description of $S H$ and $S V$ waves) and represents what would be seen in a tomographic inversion. By comparing these two velocities with the isotropic $V_{S}$, one may note that the amplitude of heterogeneities is underestimated in tomographic studies, where seismologists only have access to the homogenized medium. Middle panel: since $V_{S H} \neq V_{S V}$, the homogenized medium displays extrinsic radial shear wave anisotropy $\xi^{*}=V_{S H}^{2} / V_{S V}^{2}$, which is due to heterogeneities much smaller than $\lambda_{h}$, the minimum wavelength of the observed seismic wavefield. Bottom panel: power spectrum of heterogeneities in the isotropic medium depicted in black in the top panel. This power spectrum varies as $1 / k$, as what is expected for the Earth's mantle.

In the isotropic case, when $L=N$, we simply have $V_{S H}=V_{S V}$ and $\xi=1$.

Following pioneering work by Thomson (1950), Postma (1955) and Anderson (1961), Backus (1962) showed that in an inhomogeneous elastic medium, seismic waves are sensitive to heterogeneities much smaller than the minimum wavelength of the observed wavefield only in an effective way. As in Capdeville et al. (2015) and Capdeville \& Cance (2015), we define the parameter

$\epsilon_{0}=\frac{\lambda_{0}}{\lambda_{h}}$

where $\lambda_{h}$ is the minimum wavelength of the observed wavefield and can be expressed as $\lambda_{h}=V_{s_{\min }} / f_{\max }$ with $f_{\max }$ the cut-off frequency used to filter the seismograms. $\lambda_{h}$ will be hereafter referred to as the wavelength of homogenization. $\lambda_{0}$ is a threshold wavelength that defines the value below which all scales are considered as small scales. In this paper, we take $\epsilon_{0}=0.5$, meaning that seismic waves are sensitive to heterogeneities smaller than half the homogenization wavelength only in an effective way.

To study how much extrinsic anisotropy can be induced at large scale by the isotropic laminated structure, we use Backus' homogenization technique (Backus 1962) to describe the effective medium, that is, the long-wavelength equivalent. In the case of an isotropic horizontally layered medium, that is, VTI medium, Backus (1962) showed that the long-wavelength equivalent is a smoothly varying medium of same nature. For parameters concerning shear wave velocities, the smooth equivalent medium is then simply described by the arithmetic and harmonic spatial averages of elastic parameters $N$ and $L$ :

$N^{*}=\langle N\rangle$

$L^{*}=\langle 1 / L\rangle^{-1}$

and the effective density $\rho^{*}$ is given by the arithmetic mean of the local density:

$\rho^{*}=\langle\rho\rangle$

The symbol * refers to the long-wavelength equivalent and $\langle$.$\rangle is the$ spatial average over $\lambda_{0}$, defined in eq. (7) (Backus 1962; Capdeville et al. 2015; Capdeville \& Cance 2015). As an analogy, the relations (8) and (9) amount to estimating the equivalent spring constant when several springs are respectively in series or in parallel. In this way, long-period $S V$ waves would perceive a stack of fine layers as if these were 'in series' whereas long-period $S H$ waves would see them as if they were 'in parallel'.

In the homogenized medium, effective velocities are then simply given by:

$V_{S H}^{*}=\sqrt{N^{*} / \rho^{*}}$ 
and

$V_{S V}^{*}=\sqrt{L^{*} / \rho^{*}}$

In our case, the small-scale layered medium is isotropic, that is, $N=L$. Hence the effective anisotropy is extrinsic and simply given by the ratio of arithmetic to harmonic means of $N$ :

$\xi^{*}=\frac{N^{*}}{L^{*}}=\langle N\rangle\langle 1 / N\rangle$

As the arithmetic mean is always greater than the harmonic one, this results in having apparent radial anisotropy always greater than unity in VTI media.

In practice, the 1-D Backus' homogenization can be implemented by simply applying a low-pass Gaussian filter $\mathcal{G}(k)$ to the small-scale signal described in Section 4.1, that is, $\mathcal{G}(k)$ cancels the components with wavelengths smaller than $\lambda_{0}=\epsilon_{0} \lambda_{h}$. The elastic parameter $N$ needed for the estimation of $\xi^{*}$ in eq. (13) is obtained as follows. Given the isotropic $V_{s}$ signal and assuming a constant ratio $V_{p} / V_{s}=1.8$, the local density $\rho$ is estimated at each point of the medium following the empirical relation given in Tkalčić et al. (2006):

$\rho=2.35+0.036\left(V_{p}-3\right)^{2}$.

From there, $N$ is simply obtained by using the relation $N=\rho V_{s}^{2}$. The resulting signal is then filtered using a cut-off frequency given by the cut-off wavelength $\lambda_{0}$ (Capdeville et al. 2015). Arithmetic and harmonic means are then numerically computed as follows:

$N^{*}(z)=\langle N(z)\rangle=\mathcal{F}^{-1}[\mathcal{F}(N(z)) \times \mathcal{G}(k)]$

$L^{*}(z)=\left\langle\frac{1}{N(z)}\right\rangle^{-1}=\left(\mathcal{F}^{-1}\left[\mathcal{F}\left(\frac{1}{N(z)}\right) \times \mathcal{G}(k)\right]\right)^{-1}$

where $\mathcal{F}$ and $\mathcal{F}^{-1}$ are the Fourier transform and the inverse Fourier transform, respectively. Here $z$ refers to the spatial distance along the vertical axis and $k$ is the wavenumber. An example of a homogenized signal is shown in Fig. 2 for 5 per cent of heterogeneities (i.e. $\mathrm{d} V / V_{0}=0.05$ ) and $\lambda_{h}=200 \mathrm{~km}$.

\subsection{Quantifying the level of extrinsic anisotropy}

\subsubsection{Analytical solution}

The initial medium is isotropic so we have $N=L$ and the local extrinsic anisotropy $\xi^{*}$ is given by eq. (13) for a given homogenization wavelength $\lambda_{h}$. Let us write the parameter $N$ as $N=N_{0}+d N$ with $\langle d N\rangle=0$. Eq. (13) then becomes:

$\xi^{*}=\left\langle N_{0}+d N\right\rangle\left\langle\frac{1}{N_{0}+d N}\right\rangle$

For small heterogeneities $d N / N_{0} \rightarrow 0$, therefore since $\langle d N\rangle=0, \xi^{*}$ can be approximated to the second order by:

$$
\begin{aligned}
\xi^{*} & \approx 1+\frac{\left\langle d N^{2}\right\rangle}{N_{0}^{2}} \\
& \approx 1+\frac{\sigma_{\lambda_{0}}^{2}}{N_{0}^{2}}
\end{aligned}
$$

where $\sigma_{\lambda_{0}}^{2}=\left\langle d N^{2}\right\rangle$ is the variance of $N$ for wavelengths smaller than $\lambda_{0}$, that is the variance of heterogeneities that get smoothed out by the averaging process. This is interesting as we see here how the heterogeneities that cannot be resolved by seismic waves directly map into effective anisotropy, at least here in the 1-D case. This result is general and independent of the power spectrum of the signal.

Now considering the signal $N$ constructed using (2) with $\gamma=1$ and defining $k_{\min }=2 \pi / \lambda_{\max }$ and $k_{\max }=2 \pi / \lambda_{\min }$, we can write the power spectrum of $N$ :

$\left\{\begin{array}{lll}s_{N}(k)=S_{0} / k_{\min } & \text { for } & 2 \pi / H<k<k_{\min } \\ s_{N}(k)=S_{0} / k & \text { for } & k_{\min }<k<k_{\max } \\ s_{N}(k)=S_{0} k_{\max } / k^{2} & \text { for } & k_{\max }<k\end{array}\right.$.

The variance $\sigma_{N}^{2}$ of the signal $N$ can be computed using Parseval's identity:

$$
\begin{aligned}
\sigma_{N}^{2} & =\int_{0}^{+\infty} S_{N}(k) d k \\
& =\int_{2 \pi / H}^{k_{\min }} S_{0} \frac{1}{k_{\min }} d k+\int_{k_{\min }}^{k_{\max }} S_{0} \frac{1}{k} d k+\int_{k_{\max }}^{+\infty} S_{0} \frac{k_{\max }}{k^{2}} d k \\
& \approx S_{0}\left[2-\frac{\lambda_{\max }}{H}+\ln \left(\frac{\lambda_{\max }}{\lambda_{\min }}\right)\right] .
\end{aligned}
$$

In the same way, the variance $\sigma_{\lambda_{0}}^{2}$ of small-scale heterogeneities that cannot be resolved by seismic waves represents the part of the spectrum that is below $\lambda_{0}$ and can be expressed as:

$$
\left\{\begin{array}{lll}
\sigma_{\lambda_{0}}^{2} \approx S_{0}\left[2-\frac{\lambda_{\max }}{\lambda_{0}}+\ln \left(\frac{\lambda_{\max }}{\lambda_{\min }}\right)\right] & \text { if } & \lambda_{0}>\lambda_{\max } \\
\sigma_{\lambda_{0}}^{2} \approx S_{0}\left[1+\ln \left(\frac{\lambda_{0}}{\lambda_{\min }}\right)\right] & \text { if } & \lambda_{\min }<\lambda_{0}<\lambda_{\max } . \\
\sigma_{\lambda_{0}}^{2} \approx S_{0} \frac{\lambda_{0}}{\lambda_{\min }} & \text { if } & \lambda_{0}<\lambda_{\min }
\end{array}\right.
$$

Considering eq. (17), (19) and (20), we can eliminate $S_{0}$ and obtain, assuming $\lambda_{\min }<\lambda_{0}<\lambda_{\max }$ :

$\xi^{*} \approx 1+\left(\frac{\sigma_{N}}{N_{0}}\right)^{2} \frac{1+\ln \left(\frac{\lambda_{0}}{\lambda_{\min }}\right)}{2-\frac{\lambda_{\max }}{H}+\ln \left(\frac{\lambda_{\max }}{\lambda_{\min }}\right)}$

and in the case where $\lambda_{\min } \ll \lambda_{0} \ll \lambda_{\max }$ :

$\xi^{*} \approx 1+\left(\frac{\sigma_{N}}{N_{0}}\right)^{2} \frac{\ln \left(\frac{\lambda_{0}}{\lambda_{\min }}\right)}{\ln \left(\frac{\lambda_{\max }}{\lambda_{\min }}\right)}$

Moreover, since we have $N=\rho V_{s}^{2}$, a constant ratio $V_{p} / V_{s}$ and the empirical relation between $\rho$ and $V_{p}$ given by eq. (14), we can show that $\sigma_{N} / N_{0}$ and $\sigma_{V} / V_{0}$ are mostly proportional with $\sigma_{N} / N_{0} \approx$ $2.8 \sigma_{V} / V_{0}$. Therefore, we analytically show here that given a minimum and maximum wavelengths of heterogeneities in an isotropic layered medium, $\lambda_{\min }$ and $\lambda_{\max }$, the level of extrinsic anisotropy varies as the square of the heterogeneities. The extrinsic anisotropy also increases with the logarithm of $\lambda_{0}$, the wavelength over which heterogeneities are smoothed out, meaning that for a given $\epsilon_{0}$, it increases as the logarithm of the homogenization wavelength $\lambda_{h}=\lambda_{0} / \epsilon_{0}$, which is the minimum wavelength of the wavefield we consider.

\subsubsection{Numerical tests}

In our simple experiments, the spatial wavelengths $\lambda_{\min }$ and $\lambda_{\max }$ are fixed: $\lambda_{\min }=500 \mathrm{~m}$ and $\lambda_{\max }=H=1000 \mathrm{~km}$, insuring we are in 


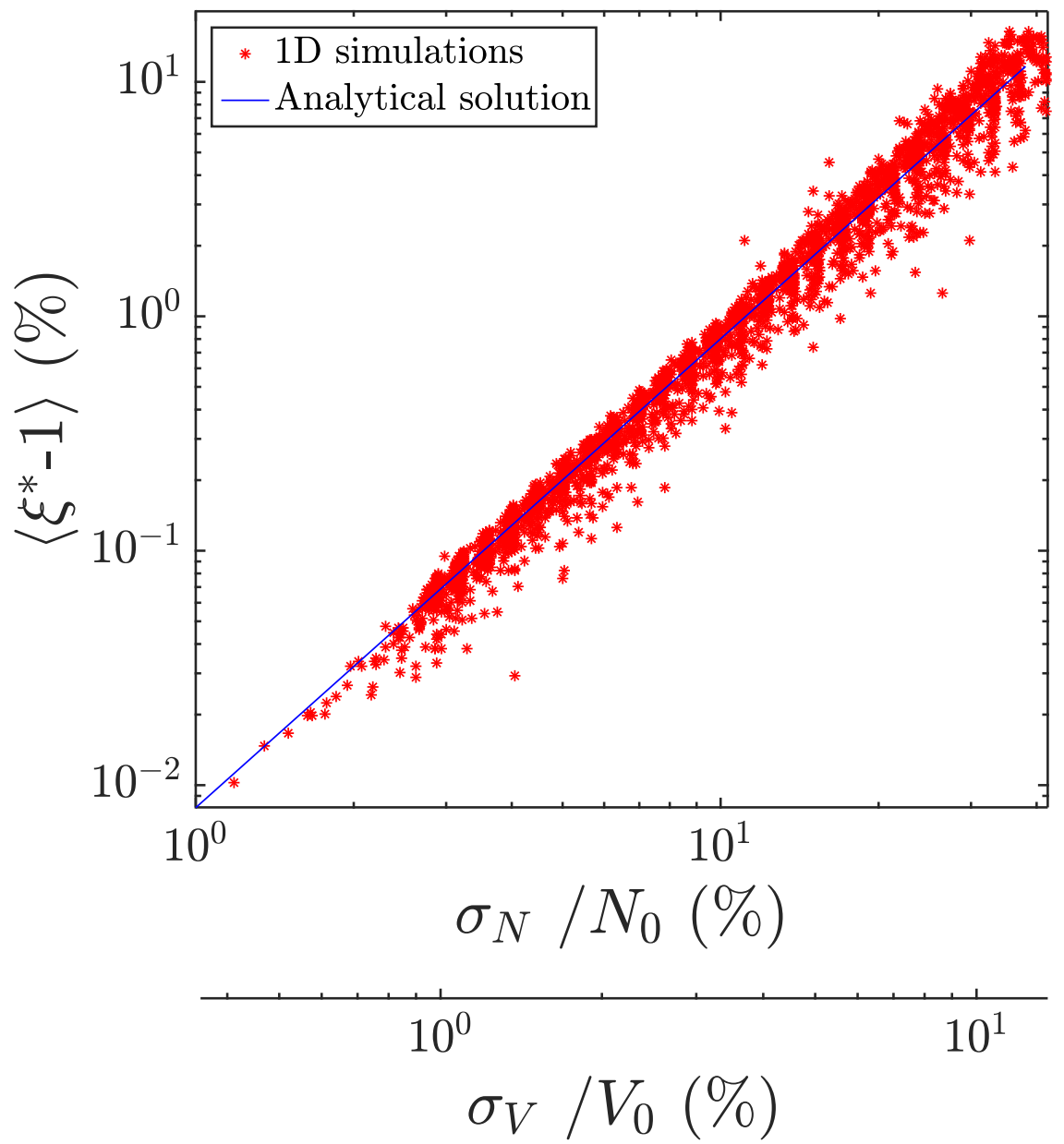

Figure 3. The experiment showed in Fig. 2 is carried out for different realizations of the isotropic layered medium (red stars). For each realization, the medium is given a different level of heterogeneities $\mathrm{d} V / V_{0}$ corresponding to a given standard deviation in percent, in terms of the $N$ parameter or in terms of shear wave velocity, respectively $\sigma_{N} / N_{0}$ or $\sigma_{V} / V_{0}$. The homogenization is done here for a wavelength $\lambda_{h}=200 \mathrm{~km}$. The level of extrinsic radial anisotropy is expressed in percent as the average of $\xi^{*}-1$ over the entire medium. The analytical relation between the level heterogeneities and that of effective anisotropy is plotted in blue.

the case where $\lambda_{\min } \ll \lambda_{0} \ll \lambda_{\max }$. In this paper, the $\epsilon_{0}$ parameter is fixed at 0.5 . As shown in eq. (22), the level of extrinsic anisotropy is then only dependent on two parameters: the level of heterogeneities and $\lambda_{h}=\lambda_{0} / \epsilon_{0}$, with $\lambda_{0}$ the wavelength over which heterogeneities are averaged.

Fig. 2 shows an example of how much extrinsic anisotropy can be induced by horizontal layering. The 1-D signal depicted in black in the top panel was obtained following the procedure described in the modelling Section 4.1 for a level of heterogeneities $\mathrm{d} V / V_{0}=5$ per cent. It was homogenized following Backus' theory for a wavelength of homogenization $\lambda_{h}=200 \mathrm{~km}$. For a shear wave propagating at $V_{s}=4.5 \mathrm{~km} \mathrm{~s}^{-1}$, this corresponds to a period of $44 \mathrm{~s}$. This homogenized medium represents what would be observed in a tomographic study of the original small-scale signal. At this point, one may note that the amplitude of heterogeneities accessible by seismic analysis, either $V_{S H}$ or $V_{S V}$, is largely underestimated. Indeed, assuming $\lambda_{\min }<\lambda_{0}<\lambda_{\max }$, heterogeneities are reduced by a factor equivalent to

$$
\left(\frac{\sigma_{\lambda_{0}}}{\sigma_{N}}\right)^{2} \approx \frac{1+\ln \left(\frac{\lambda_{0}}{\lambda_{\min }}\right)}{2-\frac{\lambda_{\max }}{H}+\ln \left(\frac{\lambda_{\max }}{\lambda_{\min }}\right)}
$$

and in the case where $\lambda_{\min } \ll \lambda_{0} \ll \lambda_{\max }$, heterogeneities are reduced by

$$
\left(\frac{\sigma_{\lambda_{0}}}{\sigma_{N}}\right)^{2} \approx \frac{\ln \left(\frac{\lambda_{0}}{\lambda_{\min }}\right)}{\ln \left(\frac{\lambda_{\max }}{\lambda_{\min }}\right)}
$$

i.e. $\sigma_{\lambda_{0}}=0.83 \sigma_{N}$ with the chosen numerical values. Moreover, the effective medium is anisotropic with $V_{S H} \neq V_{S V}$, and the extrinsic radial anisotropy $\xi^{*}$ is about 2 per cent along the whole signal.

To better investigate the relation between the level of heterogeneities, the frequency band used to image these heterogeneities and the level of mapped artificial radial anisotropy $\xi^{*}$, we successively varied $\mathrm{d} V / V_{0}$ and $\lambda_{h}$ and retrieved the extrinsic anisotropy as predicted from Backus' theory. Results are shown for $\lambda_{h}=200 \mathrm{~km}$ on the scatter plot in Fig. 3, where the average level of extrinsic radial anisotropy over the entire medium is plotted against the amplitude of heterogeneities, for a range expected in the Earth's mantle (see Section 4.1). Clearly, it confirms that the level of extrinsic anisotropy increases with the square of the level of heterogeneities, as expected from eq. (22). In addition, it is noteworthy that at shallow depths, if small-scale heterogeneities reach 10 per cent (Stixrude \& 


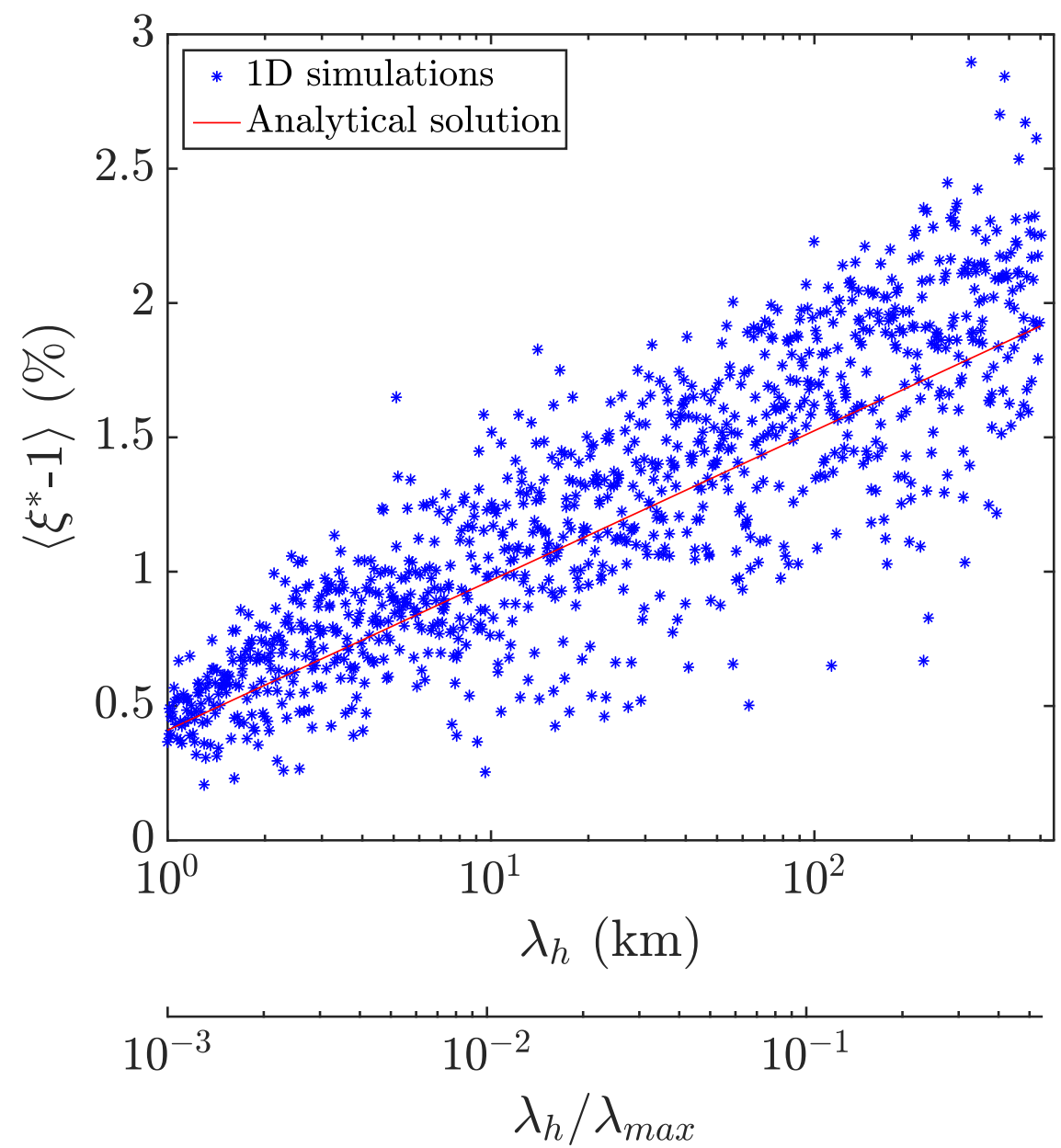

Figure 4. The experiment showed in Fig. 2 is carried out for different realizations of the isotropic layered medium (blue stars). For each realization, the medium is given a level of heterogeneities of 5 per cent and is homogenized for a given $\lambda_{h}$. The level of extrinsic radial anisotropy is expressed in percent as the average of $\xi^{*}-1$ over the entire medium. The analytical relation between the homogenization wavelength and that of effective anisotropy is plotted in red. The ratio of the homogenization wavelength over the length of the signal, in our case, the scale order of the mantle, is also displayed in the abscissa axis.

Jeanloz 2007; Xu et al. 2008), up to 8 per cent of extrinsic $S$-wave radial anisotropy is expected in this case.

Moreover, Fig. 4 illustrates that, as predicted by eq. (22), the level of extrinsic anisotropy increases as the logarithm of the homogenization wavelength $\lambda_{h}$.

\section{EXTRINSIC ANISOTROPY IN 2-D ISOTROPIC MIXING MEDIA}

\subsection{2-D velocity models based on geodynamics}

In this section we extend our study to 2-D models designed from geodynamic modelling. As illustrated by Fig. 5, they are created by numerically deforming an initial anomaly within a convecting incompressible fluid. At the end of the simulation (last panel of Fig. 5), the pattern is intended to mimic a binary medium in agreement with the marble-cake model (Allegre \& Turcotte 1986).

First we define the shape and the size of the anomaly: in our case, we begin with a circular anomaly at the centre of a box. Inside the box, the incompressible flow has horizontal and vertical velocities

$$
\left\{\begin{array}{l}
v_{y}=\frac{\partial \Psi}{\partial z} \\
v_{z}=-\frac{\partial \Psi}{\partial y}
\end{array}\right.
$$

where $\Psi$ is a time dependent stream function. We choose arbitrarily a stream function mimicking convection with the form

$\Psi(y, z, t)=\sin (\pi z)[\sin (2 \pi y)+a(t) \sin (3 \pi y)+b(t) \sin (4 \pi y)]$

which guaranties the appropriate boundary conditions,

$\begin{cases}v_{y}=0 & \text { for } y=0 \text { or } y=1 \\ v_{z}=0 & \text { for } z=0 \text { or } z=1\end{cases}$

and $a(t)$ and $b(t)$ are sinusoidal functions of time allowing the convection to be chaotic.

Given this velocity field, the points defining the contour of the anomaly are advected through an iterative procedure following a Runge-Kutta scheme with adjustable time stepping (Press et al. 1992). To better define the contour of the anomaly, new points can be added at each iteration using a spline interpolation (Press et al. 1992) where the curvature is important or the distance between points too large. Note that the length of the contour and the number of points defining this contour increase exponentially with time, and the duration time of the simulation is therefore limited. The procedure is stopped when the obtained pattern is representative of the marble-cake model as in the last panel of Fig. 5. Fig. 6 

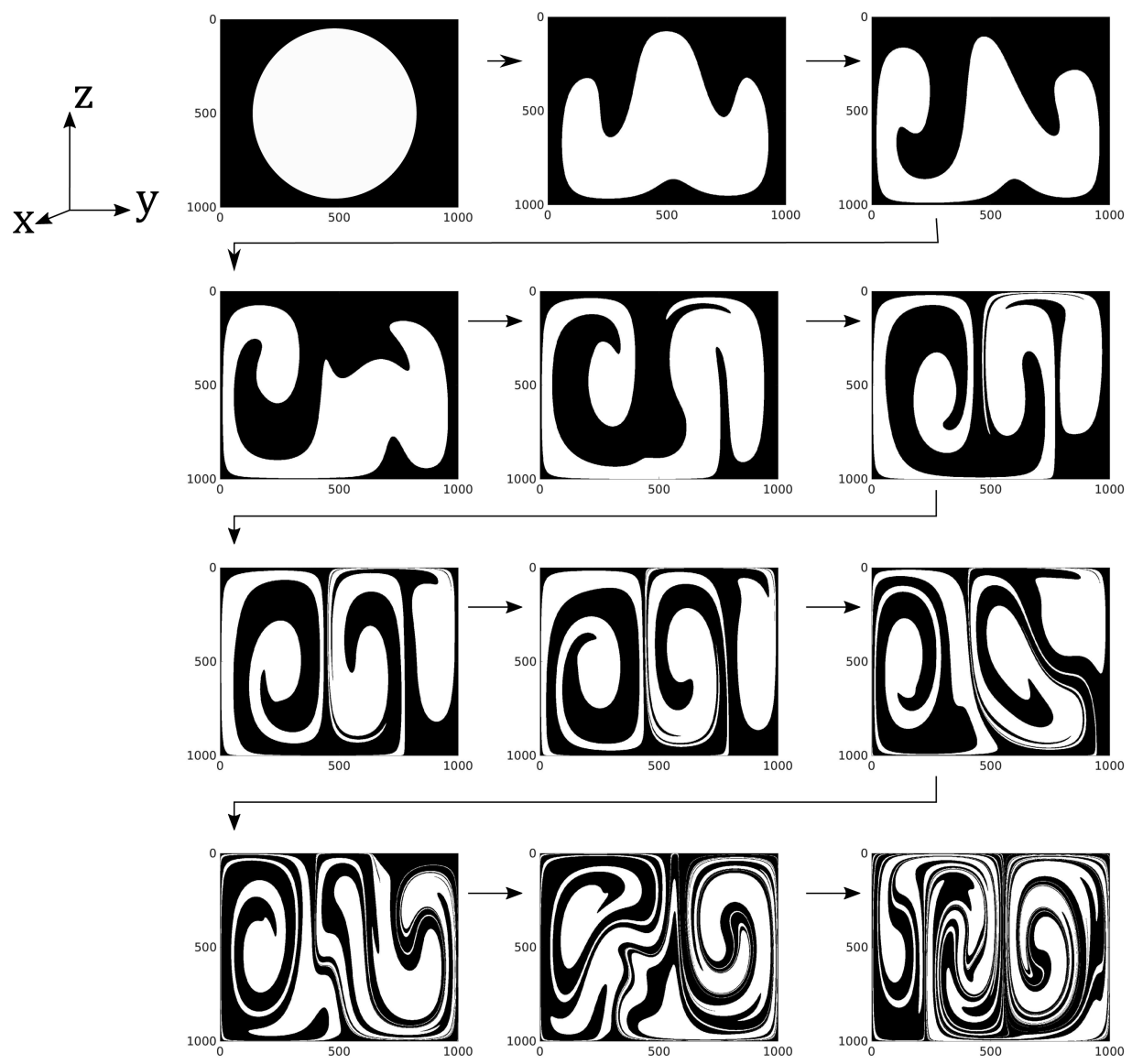

Figure 5. The 2-D models are designed by mixing a circular anomaly by chaotic convection of an incompressible fluid in a $1000 \times 1000 \mathrm{~km}{ }^{2}$ box. The final pattern is representative of a binary medium in agreement with the marble-cake model.

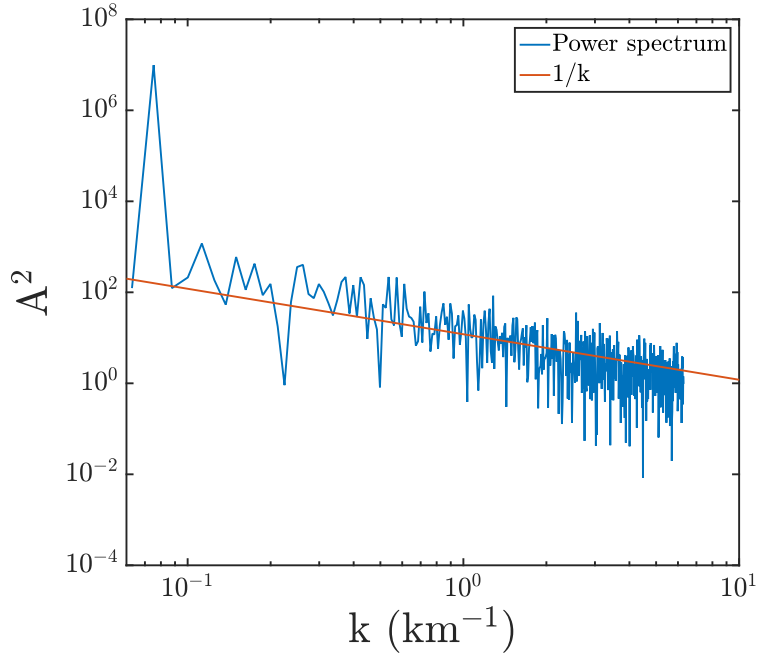

(a)

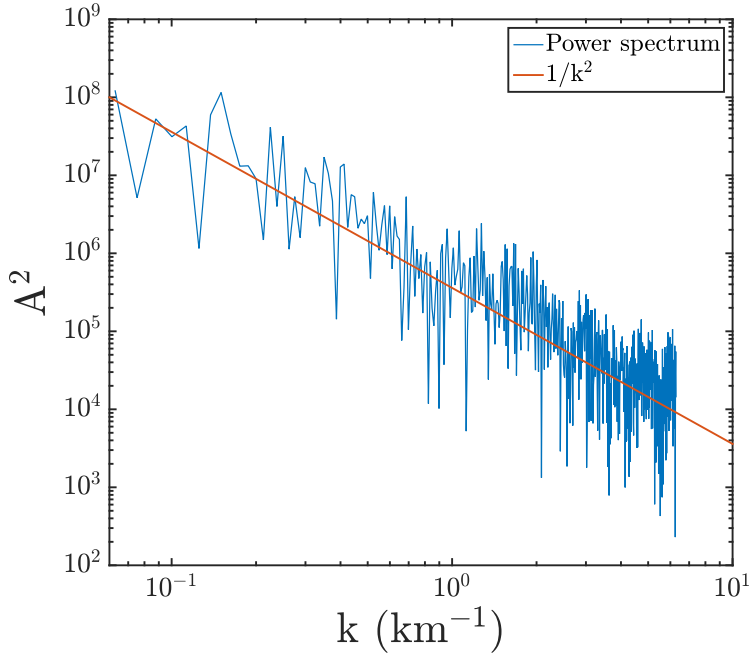

(b)

Figure 6. Power spectra of heterogeneities in our 2-D marble-cake patterns. A: amplitude of the spectrum; $k$ : wavenumber. (a) Power spectrum of a 1-D profile extracted from the 2-D marble-cake pattern. (b) 1-D section of the 2-D power spectrum of the mixing pattern.

shows that a 1-D profile extracted from our 2-D patterns has approximately a $1 / k$ spectrum, while a $1-\mathrm{D}$ section of the $2-\mathrm{D}$ power spectrum exhibits a $1 / k^{2}$ decay. This proves that our 2-D media are scale invariant (Klimeš 2002) as what is expected for the mantle in the marble-cake model.
The 2-D media, all derived from the marble-cake pattern shown in the last panel of Fig. 5, are then assigned a constant isotropic seismic velocity for each unit. Given a reference shear wave velocity for the black unit, $V_{S_{\max }}=4.5 \mathrm{~km} \mathrm{~s}^{-1}$, the $S$-wave velocity in the white unit $V_{S_{\min }}$ can be calculated for a chosen percentage of heterogeneities 
$\mathrm{d} V / V_{0}$ following eq. (1). Similarly to the 1-D case, we assume a constant ratio $V_{p} / V_{s}=1.8$, allowing to estimate the local density $\rho$ at each point of the medium following the empirical relation given in eq. (14). In addition to the density, the elastic tensor $C_{i j}$ is needed at each point to fully describe the medium. In the isotropic case, only two parameters are required to describe the $C_{i j}$ elastic tensor: $\lambda$ and $\mu$, the Lamé's coefficients with

$\lambda=\rho\left(V_{p}^{2}-2 V_{s}^{2}\right)$

$\mu=\rho V_{s}^{2}$

Using Kelvin notation, the isotropic elastic tensor can therefore be expressed at each point as:

$$
C_{i j}=\left(\begin{array}{cccccc}
\lambda+2 \mu & \lambda & \lambda & 0 & 0 & 0 \\
\lambda & \lambda+2 \mu & \lambda & 0 & 0 & 0 \\
\lambda & \lambda & \lambda+2 \mu & 0 & 0 & 0 \\
0 & 0 & 0 & 2 \mu & 0 & 0 \\
0 & 0 & 0 & 0 & 2 \mu & 0 \\
0 & 0 & 0 & 0 & 0 & 2 \mu
\end{array}\right)
$$

\subsection{The fast Fourier homogenization}

To get the long-wavelength equivalents of the 2-D marble-cake media described in the previous section and mimic the best tomographic image that seismologists would be able to retrieve, we use the 3-D Fast Fourier Homogenization technique developed by Capdeville et al. (2015). Contrary to the Backus' homogenization technique used in the 1-D layered case (Backus 1962), the Fast Fourier Homogenization does not allow to express any analytical upscaling relations in 2-D but it provides the local elastic tensor and the local density at each point of the effective medium. This medium is equivalent for the wave equation to the small-scale initial 2-D medium but it is smoothed down to the minimum wavelength of the observed wavefield. This wavelength, referred to as $\lambda_{h}$, the wavelength of homogenization, is the minimum wavelength of the observed wavefield and is associated to $f_{\max }$, the maximum frequency of the data.

Note that to retrieve the smooth equivalents of our 2-D media we could have used two 2-D homogenization codes, one for the SH case (Guillot et al. 2010) and one for the $P-S V$ system (Capdeville et al. 2010b), but that for more convenience we chose to extend our 2-D mantle-like models in the third direction and only use the 3-D Fast Fourier Homogenization, as these two methods give the same result.

\subsection{Quantifying extrinsic anisotropy in 2-D}

In the general case, the homogenized elastic tensor is complex as it displays 21 independent coefficients. The definition of anisotropy in such a medium is not as simple as in the VTI case, where the level of $S$-wave radial anisotropy can be described with a single parameter $\xi$. To quantify the level of anisotropy from a full tensor described by 21 parameters, one way would be to project this tensor onto a hexagonally symmetric one and measure the level of anisotropy in this projected tensor. This can be done following Browaeys \& Chevrot (2004), who showed how the full tensor can be decomposed into a sum of orthogonal tensors belonging to the different symmetry classes (i.e. isotropic, hexagonal, orthorhombic, etc.). Another way to quantify anisotropy would be to azimuthally average the tensor, in order to produce an effective VTI model as seen by surface waves (Montagner \& Nataf 1986). However, our 2-D patterns (in [yz]) have been extended in the third dimension $x$ and no variation occurs along the direction $x$. In such a 2.5-D medium, an azimuthal average around the vertical axis $z$ may not be meaningful. We then adopt a practical approach, and simply quantify the level of effective radial anisotropy $\xi^{*}$ as the ratio between 2 effective elastic coefficients:

$\xi^{*}=\frac{C_{66}^{*}}{C_{55}^{*}}$

with

$C_{66}^{*}=\rho^{*} V_{S H^{\prime}}^{2}$

and

$C_{55}^{*}=\rho^{*} V_{S V^{\prime}}^{2}$

Here $V_{S H^{\prime}}$ refers to the velocity of an $S$-wave propagating horizontally and polarized horizontally, that is, along the $y$ axis, and $V_{S V^{\prime}}$ refers to the velocity of an $S$-wave travelling vertically and polarized the same way (see Fig. 7). These two types of waves have the same direction of polarization, along the extended dimension $x$ : the only difference is their direction of propagation. In the case of a VTI medium, our measure of anisotropy yields the parameter $\xi^{*}$, always greater than one: the level of radial anisotropy is said to be 'positive' and $V_{S H^{\prime}}>V_{S V^{\prime}}$. On the contrary, in the case of an hexagonal symmetric medium with horizontal axis (HTI medium), with axis of symmetry $y$, our measure of $\xi^{*}$ is less than one and the level of anisotropy is 'negative', with $V_{S V^{\prime}}>V_{S H^{\prime}}$.

\subsection{Relation between level of heterogeneities and extrinsic anisotropy}

Fig. 8 shows the shear wave velocity and the anisotropy for a 2-D pattern with 10 percent of heterogeneities between the black and white units (i.e. $\mathrm{d} V / V_{0}=0.05$ ). The box size is $1000 \times 1000 \mathrm{~km}^{2}$, and the size of pixels is $2 \times 2 \mathrm{~km}^{2}$. Hence, the dynamic range defining the spectrum is $\lambda_{\max } / \lambda_{\min }=500$. Top panels in Fig. 8 show the initial isotropic $V_{s}$ pattern before homogenization and the associated level of radial anisotropy. The middle and bottom panels show results after homogenization for 2 different wavelengths of homogenization, $\lambda_{h}=200 \mathrm{~km}$ and $\lambda_{h}=500 \mathrm{~km}$. As expected, our anisotropic parameter $\xi^{*}$ is greater than 1 where the layering is horizontal and lower than 1 where it is vertical. Note also that the larger the homogenization wavelength, the smoother the longwavelength equivalent gets.

As in the 1-D case, the relation between the level of extrinsic anisotropy and the level of heterogeneities is studied by measuring the radial anisotropy in homogenized media for different percentages $\mathrm{d} V / V_{0}$ of heterogeneities. However, in 2-D, values of anisotropy are both above and below one so the mean value of apparent anisotropy over the entire medium is very close to unity. Instead of the mean value, we choose the standard deviation of $\xi^{*}$ in percent over the entire 2-D image to represent the level of extrinsic anisotropy. Fig. 9 shows that the relation is the same as in 1-D: anisotropy increases with the square of the heterogeneities. Yet, note that since the level of anisotropy is expressed in terms of a standard deviation over the 2-D model, at some locations $\xi^{*}$ may reach values much larger than what is shown in Figs 9 and 10.

In addition, Fig. 10 shows that the dependence of $\xi^{*}$ on the wavelength of homogenization is more complex than in 1-D. 


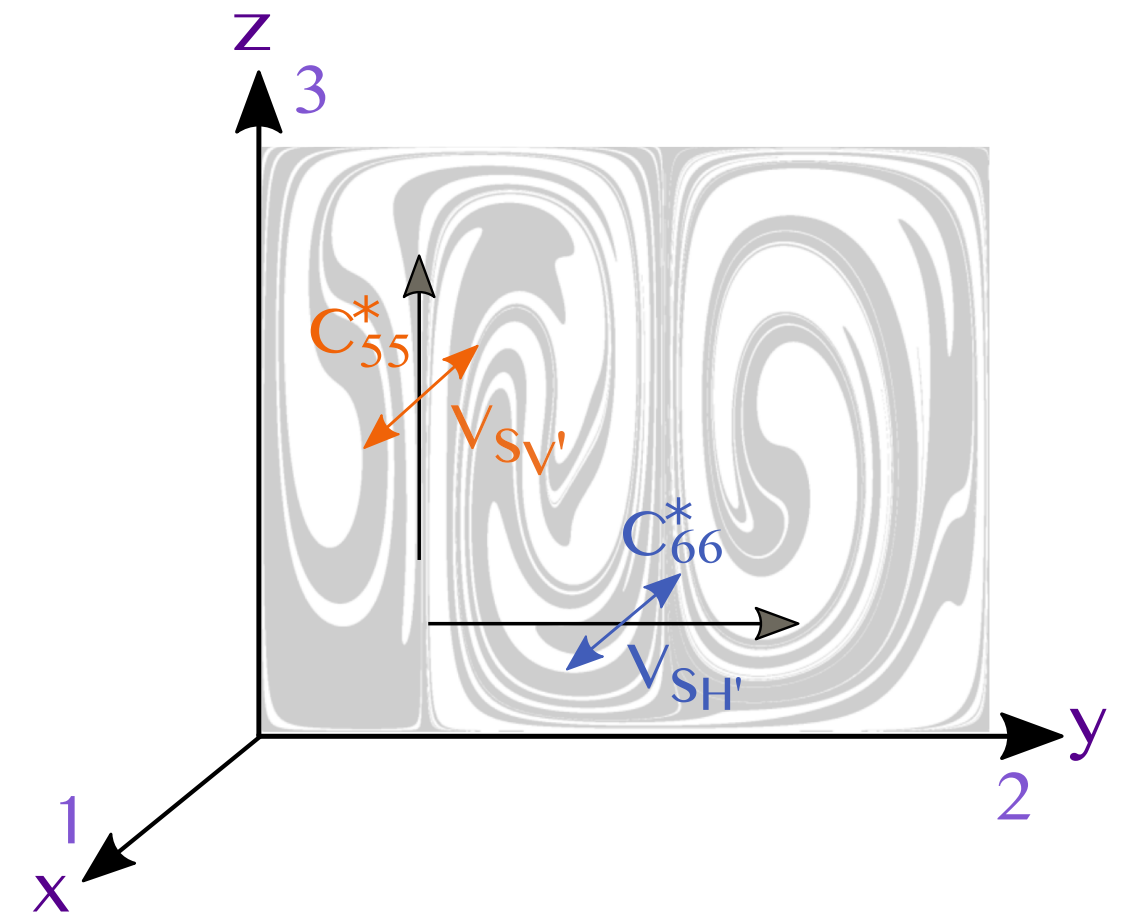

Figure 7. Coordinate system in the 2-D case. Black arrows are the direction of propagation whereas coloured arrows correspond to polarization directions. $V_{S V^{\prime}}$ is the velocity of a vertically propagating shear wave, whose polarization is along the $x$ axis, that is, the axis 1 . It is associated to the $C_{55}^{*}$ coefficient of the elastic tensor; $V_{S H^{\prime}}$ is the velocity of a shear wave propagating horizontally along the $y$ axis, with the same polarization as $V_{S V^{\prime}}$. It is associated to the $C_{66}^{*}$ coefficient of the elastic tensor.

The level of anisotropy reaches a maximum of 3.9 percent for $\sigma_{V} / V_{0}=10$ percent. This maximum is obtained for $\lambda_{h}$ of about $100 \mathrm{~km}$, that is for $\lambda_{h} / \lambda_{\max }=0.1$, where $\lambda_{\max }$ is the size of the box and represents the scale of convection cells. For a ratio $\lambda_{h} / \lambda_{\max }$ inferior to 0.1 , that is, for wavelengths which are much smaller than the size of the box, the level of anisotropy increases with $\lambda_{h}$ whereas it decreases for ratios greater than 0.1 . The value of $\lambda_{h} / \lambda_{\max }$ that maximizes the level of anisotropy does not vary with the initial level of heterogeneities. This suggests that the structure of the 2-D patterns controls the evolution of $\xi^{*}$ with the homogenization wavelength. Considering our models are representative of the whole mantle of $3000 \mathrm{~km}$ depth and given a $\lambda_{h}$ of $200 \mathrm{~km}$, a typical wavelength used in surface waves tomography, the ratio $\lambda_{h} / \lambda_{\max }$ is about 0.07 . For 10 per cent of heterogeneities, this corresponds to about 2.25 percent of extrinsic radial $S$-wave anisotropy. Therefore, in this case, the extrinsic anisotropy is non-negligible.

Contrary to the 1-D case we cannot write a rigorous analytic solution for the 2-D case. However, in the light of the numerical tests we conducted, we can easily interpret the general behaviour of extrinsic anisotropy. For small $\lambda_{h} / \lambda_{\max }$, the wavelength of homogenization is small compared to the convection cells. Therefore, around each point of the convective cell, the pattern of heterogeneity looks locally 1-D, as the folded stripes are locally parallel (see top-left corner of Fig. 8 for instance). This means that the anisotropy obeys, at each point, the eq. (22), but with a different direction of anisotropy.

At large scales (i.e. for a large $\lambda_{h} / \lambda_{\max }$ ratio), this direction becomes likely random and heterogeneities averaged over $\lambda_{h}$ have different orientations: there is no preferential direction and the averaged level of anisotropy is diminished. In this case, the level of apparent anisotropy decreases with $\lambda_{h}$ since the waves sample an incoherent layering.

In our 2-D marble-cake patterns, the directions of layering between two points can be different but are not independent. There is a typical correlation length of the convective flow, $\Delta$, such that points much closer than $\Delta$ have a similar direction of lamination while points more distant than $\Delta$ have uncorrelated directions of laminations. Visually from the two-cells situation of Fig. $5, \Delta$ is of order $300 \mathrm{~km}$. As suggested by our numerical findings, we may assume that the auto-correlation of the directions of laminations varies like a Gaussian function in the form $\exp \left(-\frac{d^{2}}{2 \Delta^{2}}\right)$ where $d$ is the distance between two points of the convective flow. The homogenization process results in averaging the model, and thus the directions of anisotropy, for wavelengths smaller than $\lambda_{0}$. This suggests that the local anisotropy given by eq. (22) is averaged by a quantity of order $\exp \left(-\frac{\lambda_{0}^{2}}{2 \Delta^{2}}\right)$ and that most probably the standard deviation of anisotropy should look like:

$\xi^{*} \approx\left(\frac{\sigma_{N}}{N_{0}}\right)^{2} \frac{\ln \left(\frac{\lambda_{0}}{\lambda_{\min }}\right)}{\ln \left(\frac{\lambda_{\max }}{\lambda_{\min }}\right)} \exp \left(-\frac{\lambda_{0}^{2}}{2 \Delta^{2}}\right)$

which is qualitatively in agreement with our findings (see Figs 9 and 10). At small scales (i.e. for $\lambda_{0} \ll \lambda_{\max }$ ), one recovers eq. (22). At large scales (i.e. for $\lambda_{h} \approx \lambda_{\max }$ ), as the local direction of anisotropy changes incoherently, the average anisotropy tends to zero.

\section{DISCUSSION AND CONCLUSIONS}

Small-scale heterogeneities in the Earth's mantle are mostly due to petrological anomalies stretched and folded during convection. This leads to a particular power spectrum of heterogeneities decreasing as $1 / k$. In this study, mantle-like isotropic models depicting such a power spectrum were created. To mimic how these models would be seen by tomography, we computed their long-wavelength equivalent thanks to Backus' theory (Backus 1962) for 1-D layered media, and the Fast Fourier Homogenization (Capdeville et al. 2015) for 2-D 

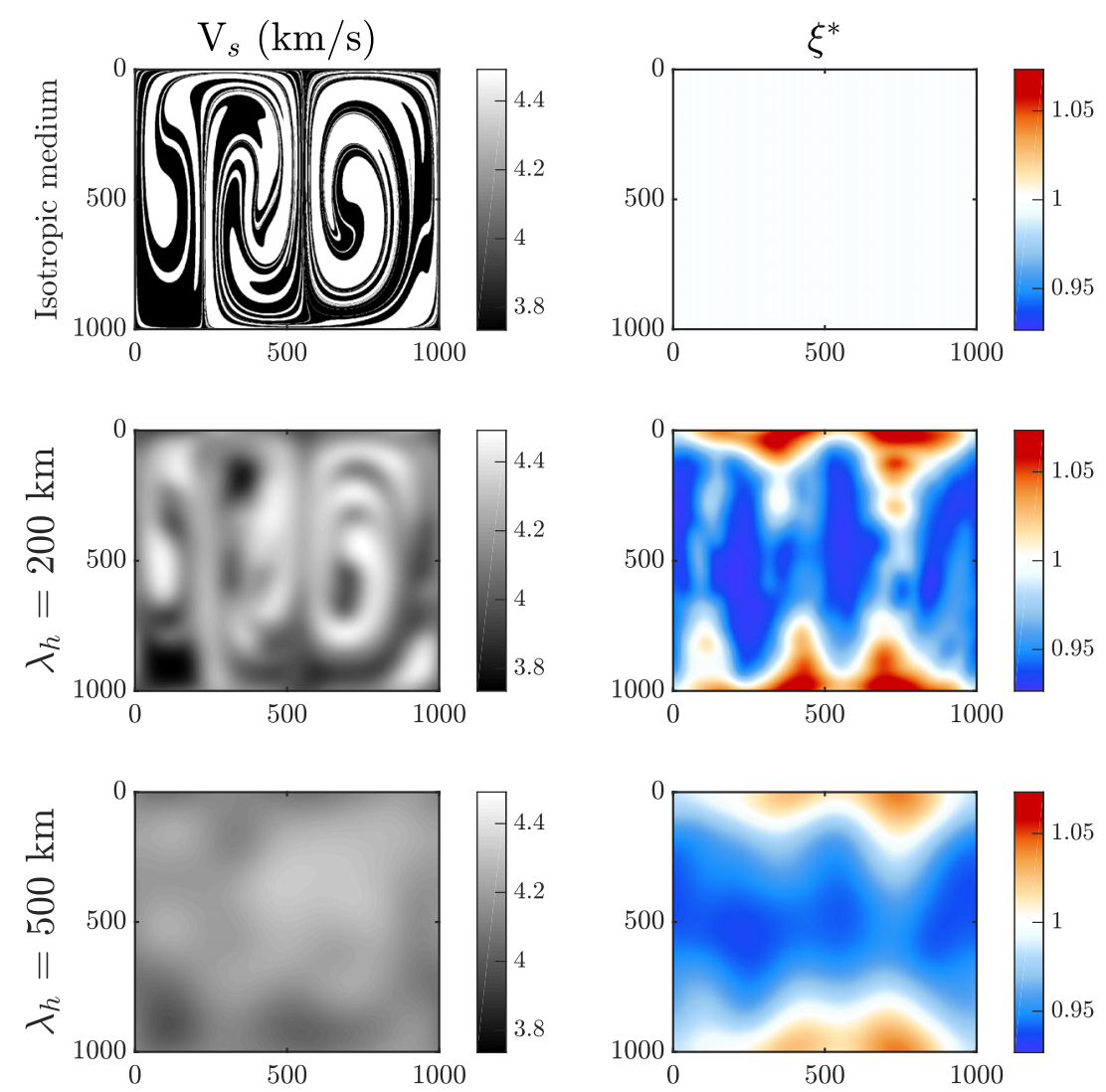

Figure 8. Homogenization of a 2-D isotropic marble-cake medium. According to eq. (1), we impose a level of heterogeneity of 10 percent between the black and white units. The resulting binary medium is upscaled for two wavelengths of homogenization $\lambda_{h}=200$ and $500 \mathrm{~km}$ following the Fast Fourier Homogenization technique (Capdeville et al. 2015). The left column displays the shear velocity and more precisely $\mathrm{V}_{S V^{\prime}}$ in the upscaled media (see Fig. 7). The right column displays the effective radial shear wave anisotropy, which is equal to one in the isotropic case, first row. Note that in the homogenized media, that is, the best possible tomographic image, the level of heterogeneities is underestimated compared to the one in the initial small-scale medium. It reaches 3.5 percent and 1.75 per cent in the medium homogenized for respectively $\lambda_{h}=200$ and $500 \mathrm{~km}$

marble-cake models. The resulting homogenized media are smooth and exhibit extrinsic radial shear wave anisotropy.

Our work shows that the level of extrinsic anisotropy observed in homogenized media increases with the square of the level of heterogeneities, both in 1-D and 2-D models. This relation was analytically proven in 1-D and verified with numerical tests in 1-D and 2-D. It is similar to that obtained by Maupin (2002) for the study of the Love-Rayleigh discrepancy. However, in her paper, Maupin (2002) also concluded that the anisotropy induced by 3-D isotropic structures is negligible compared to the observed Love-Rayleigh discrepancy. In our study, we show that extrinsic radial $S$-wave anisotropy can reach more than 3.9 per cent for heterogeneities of about 10 per cent, a typical value for chemical heterogeneities in the upper mantle. Several reasons may explain these different findings. First, our models exhibit a more realistic power spectrum of heterogeneities with a decay in $1 / k$ whereas Maupin (2002) uses smooth models with spatial correlations at distances from 20 to $100 \mathrm{~km}$. Our media exhibit smaller scales and are then more likely to produce extrinsic anisotropy. In addition, we consider the whole wave equation in the homogenization process while Maupin (2002) only uses surface waves: some effects may then have been neglected in her study. Another reason for the discrepancy between our studies may be the fact that Maupin (2002) considered 3-D structures whereas our study is based only on 1-D and 2-D media. Nevertheless, we note that our value of extrinsic anisotropy is more consistent with that estimated in PREM by Wang et al. (2015), who showed that extrinsic anisotropy can reach up to 3 per cent, corresponding to 30 percent of the observed radial anisotropy. In this way, we show that extrinsic anisotropy due to fine layering may significantly contribute to the observed anisotropy in tomographic models.

In this study, we demonstrate that a strong relation exists between heterogeneities in terms of shear wave velocity contrast and the level of extrinsic anisotropy. Hence, every factor that affects this velocity contrast may have an effect on the anisotropy. It is the case for the presence of water or partially molten zones in the mantle (e.g. Hacker et al. 2003; Kawakatsu et al. 2009; Auer et al. 2015). For instance, the full hydration of basalt and harzburgite at shallow depths near subduction zones is thought to be responsible for an increase to 20 percent and even up to 30 per cent of the heterogeneities (Hacker et al. 2003). In this case, extrinsic anisotropy might have a very significant contribution to the observed radial $S$-wave anisotropy.

We shall acknowledge that even though the models used in this work are in agreement with important observations and assumptions related to the Earth's mantle, they are still quite simple. Indeed, we did not take into account the velocity dependence on temperature and pressure, and seismic velocities in our models do not depend on depth. In the mantle, the relative velocities between basalt and peridotite are expected to generally decrease with depth (e.g. Stixrude \& Jeanloz 2007; Xu et al. 2008). In this way, assuming there is no water or partially molten zones in the mantle, the value of extrinsic anisotropy that we give should be seen as an upper bound. Another limitation to our work is that even though we used a 3-D numerical code for elastic homogenization, our patterns were in 


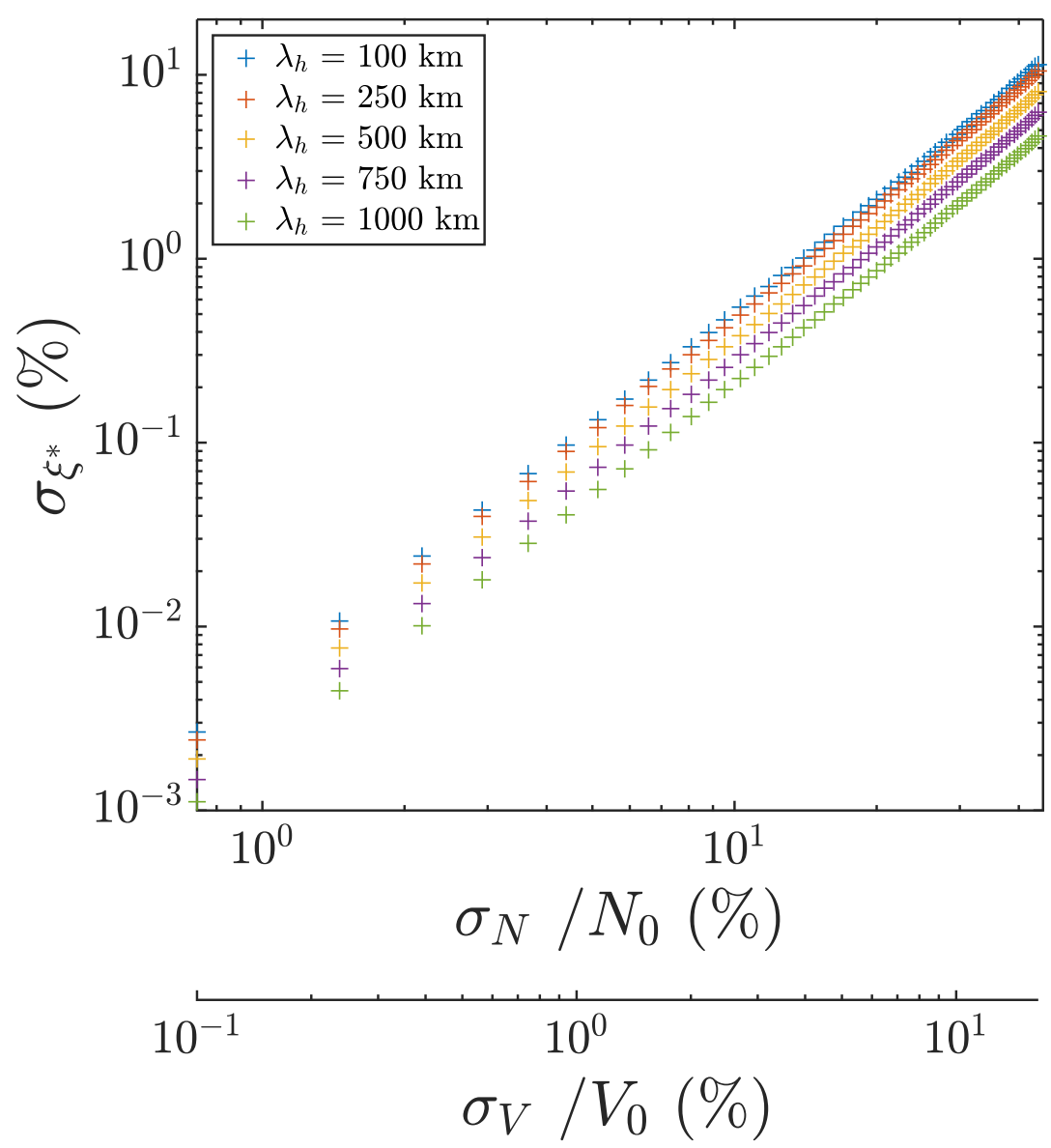

Figure 9. For several wavelengths of homogenization $\lambda_{h}$, the standard deviation of the effective radial anisotropy $\sigma_{\xi}$ is computed for 2-D isotropic media exhibiting a various range of heterogeneities. The 2-D media are derived from the single marble-cake pattern shown in the last panel of Fig. 5, which has been given different levels of heterogeneities (see Section 5.1). The heterogeneities are expressed in terms of the standard deviation in percent of the parameter $N$, $\sigma_{N} / N_{0}$, or in terms of the variations in shear waves velocities, $\sigma_{V} / V_{0}$.

two dimensions and extended along the third dimension. Hence, we did not take into account any full 3-D structural effects. Note that this is one of the reasons we chose a simple formula for apparent anisotropy in 2-D that does not involve azimuthal averaging. Future work involves testing fully 3-D geodynamical models such as StagYY (Tackley 2008).

This study is mainly a proof of concept and our main point is that a non-negligible part of the observed anisotropy in tomographic models may be extrinsic and the result of unmapped fine layering in the mantle, as previously suggested by Fichtner et al. (2013); Wang et al. (2013); Bodin et al. (2015); Wang et al. (2015). Note that similarly to this effect, unmapped velocity structure can also, at second order, be mapped as attenuation (Ricard et al. 2014). In addition, since the contrast of shear wave velocities between basalt and harzburgite is believed to be the highest in the lithosphere (e.g. Stixrude \& Jeanloz 2007; Xu et al. 2008), extrinsic anisotropy due to fine layering may particularly contribute to the observed anisotropy in the lithosphere, which is a result previously highlighted by Wang et al. (2013) and Kawakatsu (2016).

Hence one has to be careful when it comes to the interpretation of seismic anisotropy in terms of mantle deformation. Indeed, seismic anisotropy in tomography is usually assumed to be intrinsic, that is, resulting from LPO: assuming a preferential reorientation of olivine's crystalline lattices under finite strain accumulation, $\xi>1$ is usually associated with horizontal shearing whereas $\xi<1$ is assumed to indicate vertical shearing. In our 2-D models, $\xi>1$ and $\xi<1$ seem to be also related to horizontal and vertical shearing respectively. However, the interpretation is different since no LPO is involved. The shearing is here responsible for the orientation of layering in the small-scale models, that is, Shape Preferred Orientation (SPO).

Our work opens the door to new discussions about anisotropy. For example, the topography of the main discontinuities within the Earth's mantle cannot be resolved in tomographic studies, but they may have a significant effect on the level of observed radial anisotropy. Ferreira et al. (2010) have shown that lateral variations in the Moho thickness can map into radial anisotropy. Moreover, Bozdağ \& Trampert (2008); Chang \& Ferreira (2017) suggested that imperfect crustal corrections in surface waves tomographic studies have an important effect on the inferred radial anisotropy. The topography of other discontinuities, such as the transition zone discontinuities or heterogeneities at the core-mantle boundary, may also participate in the observed anisotropy (Maupin 2002; Ferreira et al. 2010). With the homogenization technique, these effects can be properly quantified, as one could measure how much a given discontinuity or its topography maps into long wavelength anisotropy. Note that in this paper, we focused on radial anisotropy, but apparent azimuthal anisotropy could also be investigated using the homogenization technique.

Finally, our study suggests that many tomographic images may be over-interpreted. Indeed, we have shown how heterogeneities observed in tomographic models are clearly underestimated. Since 


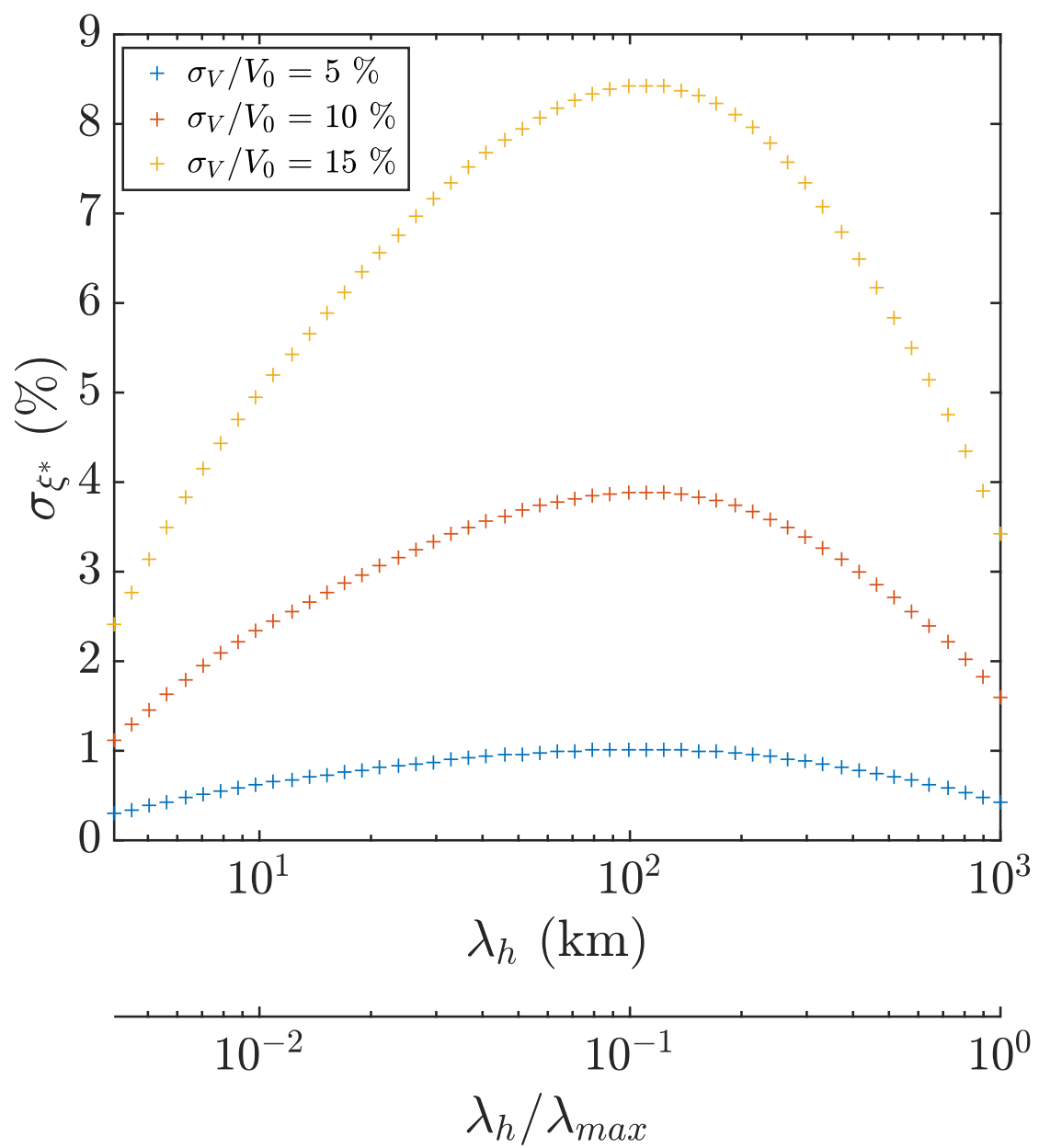

Figure 10. Standard deviation of apparent radial anisotropy $\xi^{*}$ in our 2-D isotropic media, as a function of the homogenization wavelength $\lambda_{h}$. The ratio $\lambda_{h} / \lambda_{\max }$ is also given as abscissa axis, where $\lambda_{\max }$ is the size of the box and represents the scale of convection cells. As in Fig. 9, the 2-D media are derived from the single marble-cake pattern shown in the last panel of Fig. 5 , which has been given three different levels of heterogeneities: $\sigma_{V} / V_{0}=5,10$ and 15 per cent corresponding to $\sigma_{N} / N_{0}=14.1,27$ and 38.5 per cent respectively.

seismologists work with a limited frequency band, tomographic images only represent a smooth homogenized version of the true Earth (and even worse in the case of sparse data coverage). In this way, amplitudes of seismic velocities in tomographic images may be difficult to interpret in terms of composition. Moreover, by comparing several recent global or regional models of radial anisotropy (e.g. Nettles \& Dziewoński 2006; Yuan et al. 2011; French et al. 2013; Auer et al. 2014; Chang et al. 2014, 2015; Moulik \& Ekström 2014; Zhu et al. 2017), one may note how inconsistent these models are, which makes them difficult to interpret in terms of geodynamics. These models are not only very dissimilar but they are even anticorrelated at some places. Our study suggests that it is meaningless to compare the amplitude of anisotropy in tomographic models built with different parametrizations or regularizations. This may be due in particular to the strong trade-off which exists between the level of isotropic heterogeneities in models (i.e. the spatial roughness), and the strength of anisotropy. Indeed, for the same data set, a model constructed using a coarse grid will depict more anisotropy than a model constructed using a finer grid. In the same way, for similar parametrizations, a model obtained after inverting a long period data set will be more anisotropic than a model obtained from shorter period data. The solution to the inverse problem in tomographic studies is highly non-unique and many Earth models fit the observations. By considering anisotropy as a free parameter in the inversion process, that is, as an adjustment variable, seismologists are able to better fit seismic data with smoothers models and fewer spatial parameters (Trampert \& Woodhouse 2003). The only way to compare tomographic models is to look at them at the same scale, e.g. to homogenize them up to the same wavelength. To interpret long-wavelength models in terms of deformation, new constraints on the small scales are necessary. Adding higher frequency content such as converted or reflected body waves to tomographic observations would allow to retrieve the depth of seismic discontinuities and properly distinguish the different contributions to the observed anisotropy.

\section{ACKNOWLEDGEMENTS}

We thank two anonymous reviewers for their valuable and constructive comments on the manuscript as well as the European COST action TIDES (ES1401) for discussions. This work was funded by the European Union's Horizon 2020 research and innovation programme under Grant Agreement No. 716542 and by the ANR project ANR-16-CE31-0022-01. Some of the computations were done on the Centre de Calcul Intensif des Pays de la Loire (CCIPL) computers. 


\section{REFERENCES}

Agranier, A., Blichert-Toft, J., Graham, D., Debaille, V., Schiano, P. \& Albarede, F., 2005. The spectra of isotopic heterogeneities along the midAtlantic ridge, Earth planet. Sci. Lett., 238(1-2), 96-109.

Allegre, C. \& Turcotte, D., 1986. Implications of a two-component marblecake mantle, Nature, 323, 123-127.

Anderson, D.L., 1961. Elastic wave propagation in layered anisotropic media, J. geophys. Res., 66(9), 2953-2963.

Antonsen, T.M. \& Ott, E., 1991. Multifractal power spectra of passive scalars convected by chaotic fluid flows, Phys. Rev. A, 44, 851-857.

Auer, L., Boschi, L., Becker, T.W., Nissen-Meyer, T. \& Giardini, D., 2014. Savani: a variable resolution whole-mantle model of anisotropic shear velocity variations based on multiple data sets, J. geophys. Res., 119(4), 3006-3034.

Auer, L., Becker, T.W., Boschi, L. \& Schmerr, N., 2015. Thermal structure, radial anisotropy, and dynamics of oceanic boundary layers, Geophys. Res. Lett., 42(22), 9740-9749.

Babuška, V. \& Cara, M., 1991. Seismic Anisotropy in the Earth, vol. 10 of Modern Approaches in Geophysics, Springer.

Backus, G., 1962. Long-wave elastic anisotropy produced by horizontal layering, J. geophys. Res., 67(11), 4427-4440.

Ballmer, M.D., Schmerr, N.C., Nakagawa, T. \& Ritsema, J., 2015. Compositional mantle layering revealed by slab stagnation at $1000-\mathrm{km}$ depth, Sci. $A d v$, 1(11), doi:10.1126/sciadv.1500815.

Batchelor, G., 1959. Small-scale variation of convected quantities like temperature in turbulent fluid Part 1. General discussion and the case of small conductivity, J. Fluid Mech., 5(1), 113-133.

Bensoussan, A., Lions, J.-L. \& Papanicolaou, G., 1978. Asymptotic Analysis of Periodic Structures, North-Holland Pub. Co.

Blanc, X., Le Bris, C. \& Lions, P.-L., 2007. Stochastic homogenization and random lattices, J. Math. Pures Appl., 88(1), 34-63.

Bodin, T., Capdeville, Y., Romanowicz, B. \& Montagner, J., 2015. Interpreting radial anisotropy in global and regional tomographic models, in The Earth's Heterogeneous Mantle, chap. 4, pp. 105-144, eds Khan, A. \& Deschamps, F., Springer International Publishing.

Bozdağ, E. \& Trampert, J., 2008. On crustal corrections in surface wave tomography, Geophys. J. Int., 172(3), 1066-1082.

Brandenburg, J.P. \& van Keken, P.E., 2007. Deep storage of oceanic crust in a vigorously convecting mantle, J. geophys. Res., 112(6), 1-15.

Browaeys, J.T. \& Chevrot, S., 2004. Decomposition of the elastic tensor and geophysical applications, Geophys. J. Int., 159(2), 667-678.

Capdeville, Y. \& Cance, P., 2015. Residual homogenization for elastic wave propagation in complex media, Geophys. J. Int., 200(2), 984-997.

Capdeville, Y., Guillot, L. \& Marigo, J.-J., 2010a. 1-D non-periodic homogenization for the seismic wave equation, Geophys. J. Int., 181(2), 897-910.

Capdeville, Y., Guillot, L. \& Marigo, J.-J., 2010b. 2-D non-periodic homogenization to upscale elastic media for $P-S V$ waves, Geophys. J. Int., 182(2), 903-922.

Capdeville, Y., Stutzmann, E., Nian, W. \& Montagner, J.-P., 2013. Residual homogenization for seismic forward and inverse problems in layered media, Geophys. J. Int., 194(1), 470-487.

Capdeville, Y., Zhao, M. \& Cupillard, P., 2015. Fast Fourier homogenization for elastic wave propagation in complex media, Wave Motion, 54, 170-186.

Chang, S.-J. \& Ferreira, A.M.G., 2017. Improving global radial anisotropy tomography: the importance of simultaneously inverting for crustal and mantle structure, Bull. seism. Soc. Am., 107(2), 624-638.

Chang, S.-J., Ferreira, A.M., Ritsema, J., van Heijst, H.J. \& Woodhouse, J.H., 2014. Global radially anisotropic mantle structure from multiple datasets: a review, current challenges, and outlook, Tectonophysics, 617, $1-19$.

Chang, S.-J., Ferreira, A.M.G., Ritsema, J., van Heijst, H.J. \& Woodhouse, J.H., 2015. Joint inversion for global isotropic and radially anisotropic mantle structure including crustal thickness perturbations, J. geophys. Res., 120(6), 4278-4300.
Christensen, U.R. \& Hofmann, A.W., 1994. Segregation of subducted oceanic crust in the convecting mantle, J. geophys. Res., 99(B10), $19867-19884$.

Coltice, N. \& Ricard, Y., 1999. Geochemical observations and one layer mantle convection, Earth planet. Sci. Lett., 174(1-2), 125-137.

Debayle, E. \& Ricard, Y., 2013. Seismic observations of large-scale deformation at the bottom of fast-moving plates, Earth planet. Sci. Lett., 376, 165-177.

Dellinger, J., Vasicek, D. \& Sondergeld, C., 1998. Kelvin notation for stabilizing elastic-constant inversion, Rev. Inst. Fr. Pét., 53(5), 709-719.

Durand, S., Debayle, E., Ricard, Y. \& Lambotte, S., 2016. Seismic evidence for a change in the large-scale tomographic pattern across the $\mathrm{d}^{\prime \prime}$ layer, Geophys. Res. Lett., 43(15), 7928-7936.

Farber, D.L., Williams, Q. \& Ryerson, F.J., 1994. Diffusion in $\mathrm{Mg}_{2} \mathrm{SiO}_{4}$ polymorphs and chemical heterogeneity in the mantle transition zone, Nature, 371(6499), 693-695.

Ferreira, A.M.G., Woodhouse, J.H., Visser, K. \& Trampert, J., 2010. On the robustness of global radially anisotropic surface wave tomography, J. geophys. Res., 115(B4), 1-16.

Fichtner, A., Kennett, B. \& Trampert, J., 2013. Separating intrinsic and apparent anisotropy, Phys. Earth planet. Inter., 219, 11-20.

French, S., Lekic, V. \& Romanowicz, B., 2013. Waveform tomography reveals channelled flow at the base of the oceanic asthenosphere, Science, 342(6155), 227-230.

Guillot, L., Capdeville, Y. \& Marigo, J.J., 2010. 2-D non periodic homogenization for the SH wave equation, Geophys. J. Int., 182(3), 1438-1454.

Hacker, B.R., Abers, G.A. \& Peacock, S.M., 2003. Subduction factory 1. Theoretical mineralogy, densities, seismic wave speeds, and $\mathrm{H}_{2} \mathrm{O}$ contents, J. geophys. Res., 108(B1), 2029, doi:10.1029/2001JB001127.

Helbig, K., 1994. Foundations of anisotropy for exploration seismics, in Handbook of Geophysical Exploration: Seismic Exploration, vol. 22, pp. 1-486, eds Helbig, K. \& Treitel, S., Pergamon.

Hess, H., 1964. Seismic anisotropy of the uppermost mantle under oceans, Nature, 203(4945), 629-631.

Hier-Majumder, S. \& Tauzin, B., 2017. Pervasive upper mantle melting beneath the western US, Earth planet. Sci. Lett., 463, 25-35.

Hoffman, N.R.A. \& McKenzie, D.P., 1985. The destruction of geochemical heterogeneities by differential fluid motions during mantle convection, Geophys. J. R. astr. Soc., 82(2), 163-206.

Hofmann, A.W., 1988. Chemical differentiation of the Earth: the relationship between mantle, continental crust, and oceanic crust, Earth planet. Sci. Lett., 90(3), 297-314.

Kawakatsu, H., 2016. A new fifth parameter for transverse isotropy, Geophys. J. Int., 206(1), 360-367.

Kawakatsu, H., Kumar, P., Takei, Y., Shinohara, M., Kanazawa, T., Araki, E. \& Suyehiro, K., 2009. Seismic evidence for sharp lithosphereasthenosphere boundaries of oceanic plates, Science, 324, 499-502.

Kellogg, L. \& Turcotte, D., 1987. Homogenization of the mantle by convective mixing and diffusion, Earth planet. Sci. Lett., 81(4), 371-378.

Kennett, B. \& Furumura, T., 2013. High-frequency Po/So guided waves in the oceanic lithosphere: I-Long-distance propagation, Geophys. J. Int., 195(3), 1862-1877.

Klimeš, L., 2002. Correlation functions of random media, Pure appl. Geophys., 159(7-8), 1811-1831.

Levshin, A. \& Ratnikova, L., 1984. Apparent anisotropy in homogeneous media, Geophys. J. R. astr. Soc., 76, 65-69.

Love, A., 1927. The Mathematical Theory of Elasticity, Cambridge Univ. Press.

Lowen, S.B. \& Teich, M.C., 2005. Fractal-based Point Processes, Wiley series in probability and statistics, John Wiley.

Mainprice, D., Barruol, G. \& Ben Ismail, W., 2000. The seismic anisotropy of the Earth's mantle; from single crystal to polycrystal, Geophys. Monogr., 117, 237-264.

Mancinelli, N., Shearer, P. \& Liu, Q., 2016. Constraints on the heterogeneity spectrum of Earth's upper mantle, J. geophys. Res., 121(5), 3703-3721.

Mao, Z., Fan, D., Lin, J.-F., Yang, J., Tkachev, S.N., Zhuravlev, K. \& Prakapenka, V.B., 2015. Elasticity of single-crystal olivine at high pressures and temperatures, Earth planet. Sci. Lett., 426, 204-215. 
Maupin, V., 2002. The amplitude of the Love-Rayleigh discrepancy created by small-scale heterogeneities, Geophys. J. Int., 150(1), 58-64.

Maupin, V. \& Park, J., 2007. Theory and observations-wave propagation in anisotropic media., in Treatise on Geophysics, Vol. 1, pp. 289-321, ed. Schubert, G., Elsevier.

Montagner, J.-P., 1998. Where can seismic anisotropy be detected in the Earth's mantle? In boundary layers, Pure appl. Geophys., 151(2), 223-256.

Montagner, J.-P. \& Nataf, H.-C., 1986. A simple method for inverting the azimuthal anisotropy of surface waves, J. geophys. Res., 91(B1), 511-520.

Morgan, J.P. \& Morgan, W., 1999. Two-stage melting and the geochemical evolution of the mantle: a recipe for mantle plum-pudding, Earth planet. Sci. Lett., 170(3), 215-239.

Moulik, P. \& Ekström, G., 2014. An anisotropic shear velocity model of the Earth's mantle using normal modes, body waves, surface waves and long-period waveforms, Geophys. J. Int., 199(3), 1713.

Nakagawa, T. \& Buffett, B.A., 2005. Mass transport mechanism between the upper and lower mantle in numerical simulations of thermochemical mantle convection with multicomponent phase changes, Earth planet. Sci. Lett., 230(1-2), 11-27.

Nakagawa, T. \& Tackley, P.J., 2014. Influence of combined primordial layering and recycled MORB on the coupled thermal evolution of Earth's mantle and core, Geochem. Geophys. Geosyst., 15(3), 619-633.

Nettles, M. \& Dziewoński, A.M., 2006. Radially anisotropic shear velocity structure of the upper mantle globally and beneath North America, J. geophys. Res., 113(2), 1-27.

Olson, P., Yuen, D.A. \& Balsiger, D., 1984. Mixing of passive heterogeneities by mantle convection, J. geophys. Res., 89(B1), 425-436.

Postma, G.W., 1955. Wave propagation in a stratified medium, Geophysics, 20(4), 780-806.

Press, W., Teukolsky, S., Vetterling, W. \& Flannery, B., 1992. Numerical Recipies in Fortran 77: The art of scientific programming, 2nd edn, Cambridge Univ. Press.

Ribe, N., Stutzmann, E., Ren, Y. \& van der Hilst, R., 2007. Buckling instabilities of subducted lithosphere beneath the transition zone, Earth planet. Sci. Lett., 254(1-2), 173-179.

Ricard, Y., Richards, M., Lithgow-Bertelloni, C. \& Le Stunff, Y., 1993. A geodynamic model of mantle density heterogeneity, J. geophys. Res., 98(B12), 21 895-21909.

Ricard, Y., Mattern, E. \& Matas, J., 2005. Synthetic Tomographic Images of Slabs from Mineral Physics, pp. 283-300, American Geophysical Union.

Ricard, Y., Durand, S., Montagner, J. \& Chambat, F., 2014. Is there seismic attenuation in the mantle?, Earth planet. Sci. Lett., 388, 257-264.

Sanchez-Palencia, E., 1980. Non-homogeneous Media and Vibration Theory, no. 127 in Lecture Notes in Physics, Springer.

Stixrude, L. \& Jeanloz, R., 2007. Constraints on seismic models from other disciplines - constraints from mineral physics on seis- mological models, in Treatise on Geophysics, vol. 1, pp. 775-803, ed. Schubert, G., Elsevier.

Tackley, P.J., 2008. Modelling compressible mantle convection with large viscosity contrasts in a three-dimensional spherical shell using the yinyang grid, Phys. Earth planet. Inter., 171(1), 7-18.

Tauzin, B. \& Ricard, Y., 2014. Seismically deduced thermodynamics phase diagrams for the mantle transition zone, Earth planet. Sci. Lett., 401, 337-346.

Tauzin, B., Bodin, T., Debayle, E., Perrillat, J.-P. \& Reynard, B., 2016. Multi-mode conversion imaging of the subducted Gorda and Juan de Fuca plates below the North American continent, Earth planet. Sci. Lett., 440, 135-146.

Thomson, W., 1856. Elements of a mathematical theory of elasticity, Phil. Trans. R. Soc. A, 146, 481-498.

Thomson, W., 1878. Mathematical theory of elasticity, in Encyclopaedia Britannica, 9th edn, vol. 7, chap. 10, pp. 819-825, ed. Baynes, T.S., A. \& C. Black, Edinburgh.

Thomson, W.T., 1950. Transmission of elastic waves through a stratified solid medium, J. Appl. Phys., 21(2), 89-93.

Tkalčić, H., Pasyanos, M.E., Rodgers, A.J., Gök, R., Walter, W.R. \& AlAmri, A., 2006. A multistep approach for joint modeling of surface wave dispersion and teleseismic receiver functions: implications for lithospheric structure of the Arabian Peninsula, J. geophys. Res., 111(B11), $1-25$.

Trampert, J. \& Woodhouse, J.H., 2003. Global anisotropic phase velocity maps for fundamental mode surface waves between 40 and 150s, Geophys. J. Int., 154(1), 154-165.

Vinnik, L., Farra, V. \& Romanowicz, B., 1989. Azimuthal anisotropy in the Earth from observations of SKS at GEOSCOPE and NARS broadband stations, Bull. seism. Soc. Am., 79(5), 1542-1558.

Wang, N., Montagner, J., Fichtner, A. \& Capdeville, Y., 2013. Intrinsic versus extrinsic anisotropy: the radial anisotropy in reference Earth models, Geophys. Res. Lett., 40(16), 4284-4288.

Wang, N., Montagner, J.-P., Burgos, G., Capdeville, Y. \& Yu, D., 2015. Intrinsic versus extrinsic seismic anisotropy: surface wave phase velocity inversion, C. R. Geosci., 347(2), 66-76.

Xu, W., Lithgow-Bertelloni, C., Stixrude, L. \& Ritsema, J., 2008. The effect of bulk composition and temperature on mantle seismic structure, Earth planet. Sci. Lett., 275(1-2), 70-79.

Yamazaki, D., Kato, T., Yurimoto, H., Ohtani, E. \& Toriumi, M., 2000. Silicon self-diffusion in $\mathrm{MgSiO}_{3}$ perovskite at $25 \mathrm{GPa}$, Phys. Earth planet. Inter., 119(3-4), 299-309.

Yuan, H., Romanowicz, B., Fischer, K.M. \& Abt, D., 2011. 3-D shear wave radially and azimuthally anisotropic velocity model of the North American upper mantle, Geophys. J. Int., 184(3), 1237-1260.

Zhu, H., Komatitsch, D. \& Tromp, J., 2017. Radial anisotropy of the North American upper mantle based on adjoint tomography with USArray, Geophys. J. Int., 211, 349-377. 